\title{
Die Vertiefte Überprüfung von Stauanlagen in Deutschland
}

\author{
Markus Aufleger - Maximilian Knallinger - Alexander Walter
}

Angenommen: 11. März 2021 / Online publiziert: 9. April 2021

(C) Der/die Autor(en) 2021

Zusammenfassung Stauanlagen müssen dauerhaft überwacht werden. In Deutschland werden im Zuge der Vertieften Überprüfungen in einem Zeitabstand von etwa 10-20 Jahren mit einem vergleichsweise großen Aufwand alle sicherheitsrelevanten Nachweise und Fakten dieser Bauwerke erhoben und bewertet. Die Grundlagen hierfür sind in verschiedenen Normen und Regelwerken zu finden. Auf Basis ihrer Erfahrungen in verschiedenen Projekten werden von den Autoren grundlegende Informationen und aktuelle praktische Erfahrungen dargestellt. Anhand tabellarischer Zusammenstellungen und verschiedener Beispiele werden typische Fragestellungen bei diesen sehr detaillierten Prüfungen in verkürzter Form vorgestellt. Der Schwerpunkt dieser Veröffentlichung liegt auf der Vertieften Überprüfung von Talsperren. Es wird gezeigt, dass diese Untersuchungen einen sehr hohen Aufwand erfordern. Sanierungserfordernisse müssen im Zuge dieser Überprüfungen erkannt werden.

Schlüsselwörter Talsperrensicherheit . Talsperrenüberwachung · Vertiefte Überprüfung

Univ.-Prof. Dr.-Ing. M. Aufleger ( $₫)$ Institut für Infrastruktur, Arbeitsbereich Wasserbau, Universität Innsbruck, Technikerstraße 13, 6020 Innsbruck, Österreich markus.aufleger@uibk.ac.at

DI M. Knallinger m4 Ingenieure $\mathrm{GmbH}$, Augustenstraße 10, 80333 München, Deutschland

knallinger@m4-ingenieure.de

\section{A. Walter}

Bau+Plan Ingenieurgesellschaft mbH, Dorfstraße 39, 81247 München,

Deutschland

walter@plan-gmbh.de

\section{Detailed dam safety review in Germany}

Abstract Dams must be permanently monitored. In Germany, all safety-relevant proofs, analyses and facts of these structures are collected and evaluated in the course of the in-depth inspections at intervals of about 10 years to 20 years with a comparatively large effort. The basis for this can be found in various standards and regulations. Based on their experience in various projects, the authors present basic information and current practical experience. Using tabular compilations and various examples, typical issues in these very detailed inspections are shown in a simplified form. The focus of this publication is on the in-depth review of large dams. It is shown that these investigations require a very high effort. Rehabilitation needs must be identified in the course of these reviews.

Keywords Dam Safety · Dam Surveillance $\cdot$ In-depth inspections

\section{Die fachliche Bedeutung der Vertieften Überprüfung von Stauanlagen}

Stauanlagen besitzen in der Regel ein erhebliches Gefährdungspotenzial. Ihre Lebensdauer ist sehr groß. Im Sinne einer dauerhaften Überwachung des Zustands der Bauwerke hat sich die Notwendigkeit und Sinnhaftigkeit gezeigt, in Ergänzung zu der ohnehin routinemäßig erforderlichen Bauwerksüberwachung (z.B. die kontinuierliche Messung der Sickerwasserabflüsse oder eine im Abstand von einigen Monaten immer wiederkehrende Messung der Setzungen) in deutlich größeren Zeitabständen tiefer gehende Überprüfungen der Stauanlagen vorzunehmen. Sanierungserfordernisse und sich entwickelnde Defizite bzw. Schwachstellen müssen im Zuge dieser Vertieften Überprüfungen erkannt werden. Etwaige Sanierungen oder andere weitergehende Maßnahmen müssen im Rahmen dieser Untersuchungen angeregt bzw. angestoßen werden.

\section{Rechtliche Grundlagen,} Regelwerke, Empfehlungen

\subsection{Einordnung in internationalen Kontext}

Der Betrieb, der Unterhalt, die Überwachung aber auch die wiederkehrende Überprüfung von Stauanlagen wird international - aber auch auf nationaler oder regionaler Skala - höchst unterschiedlich gehandhabt. Talsperren riegeln den Querschnitt eines Tales vollständig $a b$ und sind hinsichtlich ihrer typischen Höhen und Gefährdungspotenziale als eine Teilgruppe der Stauanlagen $\mathrm{zu}$ sehen, für welche besonders hohe Anforderungen an die Sicherheitsüberwachung zu stellen sind.

Innerhalb der International Commission on Large Dams (ICOLD) wurde in einem zeitaufwendigen Abstimmungsprozess versucht, eine gemeinsame Vorstellung der fachgerechten Überwachung von Talsperren $\mathrm{zu}$ formulieren. In ICOLD Bulletin 158 (International Commission on Large Dams 2018) sind Grundsätze der verschiedenen Ebenen der Talsperrenüberwachung zusammengestellt (Abb. 1). In einem zeitlichen Abstand von etwa 5-15 Jahren werden hier formale, tiefergehende Überprüfungen der Talsperren empfohlen.

\subsection{Gesetz zur Ordnung des Wasserhaushalts (Wasserhaushaltsgesetz - WHG)}

Zum Zeitpunkt der Erstellung grundlegender Regelwerke zur Vertieften Überprüfung (DIN 19700, DVWK-Merkblatt 231/1995 (1995)) wurde der Rahmen der Überwachung wasserbaulicher Anlagen in Deutschland noch in $\$ 21$ des im Jahre 2010 außer Kraft getretenen Wasserhaushaltsgesetzes geregelt. Seit der Neufassung des Gesetzes zur Ordnung des Wasserhaushalts (Wasserhaushaltsgesetz - WHG) ab 2009 wer- 


\section{Betriebszeit ('Lebenszeit') der Talsperre}

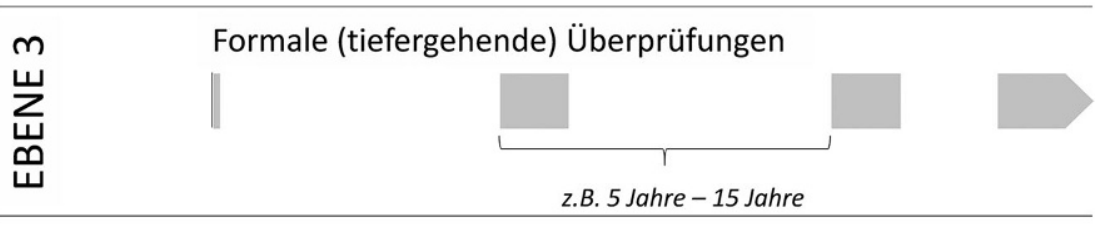

Periodische Sicherheitsüberprüfungen
Prüfen und Testen der Betriebseinrichtungen
岗

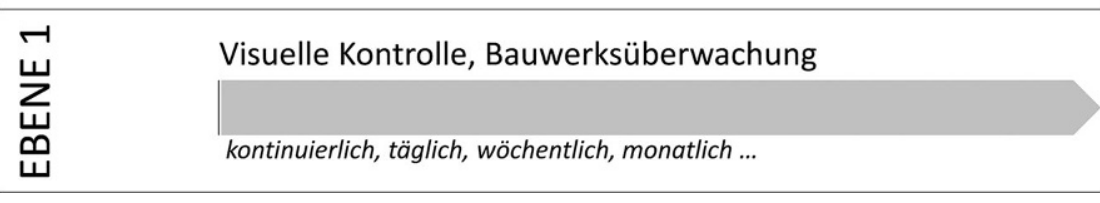

Abb. 1 Darstellung der Aktivitäten bei der Talsperrenüberwachung während der Lebenszeit einer Talsperre. (In Anlehnung an ICOLD 2018)

den diese Grundsätze in $§ 36$ (2) WHG dargestellt:

\$ 36 Anlagen in, an, über und unter oberirdischen Gewässern

(...)

(2) Stauanlagen und Stauhaltungsdämme sind nach den allgemein anerkannten Regeln der Technik zu errichten, zu betreiben und zu unterhalten; die Anforderungen an den Hochwasserschutz müssen gewahrt sein. Wer Stauanlagen und Stauhaltungsdämme betreibt, hat ihren ordnungsgemäßen Zustand und Betrieb auf eigene Kosten zu überwachen (Eigenüberwachung). Entsprechen vorhandene Stauanlagen oder Stauhaltungsdämme nicht den vorstehenden Anforderungen, so kann die zuständige Behörde die Durchführung der erforderlichen Maßnahmen innerhalb angemessener Fristen anordnen.

Hieraus ist abzuleiten, dass Talsperren und andere Stauanlagen während ihrer - typischerweise sehr langen - Lebenszeiten grundsätzlich an die allgemein anerkannten Regeln der Technik anzupassen sind.
Das Wasserhaushaltsgesetz gilt für alle Stauanlagen in Deutschland. In den relevanten Landeswassergesetzen der deutschen Bundesländer werden die Regelungen der Überwachung dieser Bauwerke fallweise nach spezifischen Größen- bzw. Klassenkriterien weiter unterteilt (Overhoff et al. 2004).

\subsection{DIN 19700 „Stauanlagen“}

In DIN 19700 „Stauanlagen“ (DIN 19700 10:2004-7 (2004); DIN 19700-11:2004-7 (2004); DIN 19700-12:2004-7 (2004); DIN 19700-13:2019-6 (2019)) finden sich verschiedene Formulierungen (Tab. 1) in Bezug auf das Erfordernis der Durchführung tiefer gehender Überprüfungen von Stauanlagen in angemessenen Zeitabständen.

Die Vertiefte Überprüfung ist als Bestandteil der Bauwerksüberwachung von Stauanlagen in Deutschland so weit institutionalisiert, dass dies auch durch die Großschreibung „Vertiefte Überprüfung“ dokumentiert wird. In vorliegender Veröffentlichung wird die Kleinschreibung „vertiefte Überprüfung" nur in Zitaten (insbesondere der DIN 19700) verwendet.
Als relevante Themengebiete der Vertieften Überprüfungen werden in DIN 19700

- die statischen Bemessungsgrundlagen,

- die hydrologischen Bemessungsgrundlagen,

- die hydraulischen Bemessungsgrundlagen,

- die betrieblichen Vorgaben und

- das Überwachungskonzept

genannt, ohne jedoch weiter auf den tatsächlich erforderlichen Umfang einzugehen. Zur Klarstellung der tatsächlich in der deutschen Norm DIN 19700 $\mathrm{zu}$ findenden konkreten Regelungen sind in Tab. 1 die relevanten Formulierungen zitiert.

Konkrete Zeitabstände der Vertieften Überprüfungen werden nur in der aktualisierten DIN 19700-13:2019-6 (2019) (Staustufen) mit etwa 10-20 Jahren vorgegeben.

\subsection{DVWK- und DWA-Regelwerke bzw. Empfehlungen}

Der Begriff der „vertieften Überprüfung“ wurde erstmals im DVWK-Merkblatt 231 (1995) eingeführt und definiert (Tab. 2). Dort finden sich bereits die unter Abschn. 2.3. aufgezeigten relevanten Themengebiete (siehe oben). Es wird auch auf das mögliche Erfordernis ergänzender Gutachten hingewiesen. Für Talsperren wird ein Zeitabstand von etwa 10 Jahren beschrieben. Dieses Merkblatt wurde unter Einbindung der Fachöffentlichkeit im Rahmen eines Gelbdruckverfahrens verabschiedet.

\subsection{Weitere Veröffentlichungen}

Aus dem Kreis der Bearbeiter des DVWK-Merkblatts und der DIN 19700 wurde im Jahr 2004 ein Überblick bzw. eine weitere mögliche Untersetzung der Themengebiete bei diesen periodischen Überprüfungen veröffentlich (Overhoff et al. 2004). In einem Gliederungsbeispiel wird vorgeschlagen, im Anschluss an einen Allgemeinen Teil die Ergebnisse der Vertieften Überprüfung folgendermaßen zu strukturieren:

A. Hydrologische Nachweise,

B. Hydrotechnische Nachweise,

C. Untergrund,

D. Bauwerke,

E. Betriebseinrichtungen,

F. Überwachung, 
Hier steht eine Anzeige.

Springer 
Hier steht eine Anzeige.

Springer 


\begin{tabular}{|c|c|c|c|}
\hline Teil & $\mathrm{Nr}$ & $\begin{array}{l}\text { Relevante Formulierungen in Bezug auf vertiefte Überprüfungen } \\
\text { (Zitate, fallweise gekürzt) }\end{array}$ & Anmerkung \\
\hline $\begin{array}{l}\text { 19700-10: } \\
2004-7 \\
\text { Gemeinsame } \\
\text { Festlegungen }\end{array}$ & 11 & $\begin{array}{l}\text { Die Überwachung von Stauanlagen unter Sicherheitsaspekten ist } \\
\text { während ihrer gesamten Nutzungsdauer erforderlich. Sie umfasst im } \\
\text { Wesentlichen: } \\
\text { Messungen, Beobachtungen und deren Auswertung in engen, zeitli- } \\
\text { chen Abständen; } \\
\text { Regelmäßige, zumeist jährliche Sicherheitsberichte; } \\
\text { Vertiefte Überprüfungen in angemessenen Zeitabständen sowie } \\
\text { gegebenenfalls in Abhängigkeit des Gefährdungspotenzials und } \\
\text { gegebenenfalls nach außergewöhnlichen Ereignissen ... } \\
\text { In der vertieften Überprüfung sind alle relevanten Sicherheitsnach- } \\
\text { weise, für die sich Veränderungen der Eingangsparameter ergeben } \\
\text { haben, mit den aktuellen gültigen Kennwerten und nach den jeweils } \\
\text { gültigen technischen Vorschriften erneut zu führen }\end{array}$ & $\begin{array}{l}\text { Hier wird der Begriff „,vertiefte Überprüfung“ eingeführt und } \\
\text { grundlegend definiert }\end{array}$ \\
\hline \multirow[t]{3}{*}{$\begin{array}{l}\text { 19700-11: } \\
2004-7 \\
\text { Talsperren }\end{array}$} & 10.3 & $\begin{array}{l}\text { Die Gebrauchstauglichkeit und die Funktionsfähigkeit der Betriebsein- } \\
\text { richtungen der Talsperre sind in der Regel unter Betriebsbedingungen } \\
\text { nach einem in der Betriebsvorschrift vorgegebenen Zeitraum zu } \\
\text { überprüfen. Zur Überprüfung gehören insbesondere Funktionsprüfun- } \\
\text { gen an Verschlüssen, Armaturen, Antrieben sowie an Mess-, Steuer } \\
\text { und Regelanlagen. Bei Erfordernis gehören zur Überprüfung auch } \\
\text { Dickemessungen an Rohrwandungen, Druck- und Fließgeschwindig- } \\
\text { keitsmessungen in Rohrleitungen und Schwingungsmessungen bei } \\
\text { Erschütterungen oder Kavitation. Zur Betriebsüberwachung gehört } \\
\text { auch die Prüfung der ausreichenden Belüftung der Räume, wie z.B. } \\
\text { Schieberkammern, Kontrollgänge, Stollen und Schächte }\end{array}$ & \multirow[t]{3}{*}{$\begin{array}{l}\text { Diese Norm gilt in Verbindung mit DIN 19700-10:2004-7. } \\
\text { In 10.3. werden grundlegende Aspekte der regelmäßigen } \\
\text { Überprüfung der Betriebseinrichtungen beschrieben, wobei } \\
\text { dort auf unterschiedliche „Erfordernisse“ eingegangen wird. } \\
\text { Die sehr detaillierten Überprüfungen (z. B. Dickemessungen } \\
\text { der Rohrwandungen) sind hier den ,Vertieften Überprüfungen“ } \\
\text { zuzuordnen. } \\
\text { 10.4. zählt die wichtigsten Themengebiete der Vertieften } \\
\text { Überprüfungen auf. } \\
\text { In } 10.5 \text { wird klargestellt, dass die Berichte zur Vertieften } \\
\text { Überprüfung als Teile des Talsperrenbuches dauerhafter } \\
\text { Bestandteil der Dokumentation der Talsperren sind }\end{array}$} \\
\hline & 10.4 & $\begin{array}{l}\text { Im Rahmen der vertieften Überprüfung sind die statischen, hydrologi- } \\
\text { schen und hydraulischen Bemessungsgrundlagen sowie die betrieb- } \\
\text { lichen Vorgaben und das Überwachungskonzept zu überprüfen. Die } \\
\text { Ergebnisse sind in Berichtsform zu dokumentieren }\end{array}$ & \\
\hline & 11.2 & $\begin{array}{l}\text { Inhalt des Talsperrenbuches } \\
\text { Überwachungsunterlagen wie ... Berichte über vertiefte Überprüfun- } \\
\text { gen }\end{array}$ & \\
\hline $\begin{array}{l}\text { 19700-12: } \\
2004-7 \\
\text { Hochwasser- } \\
\text { rückhalte- } \\
\text { becken }\end{array}$ & 10.3 & $\begin{array}{l}\text { Im Rahmen der vertieften Überprüfungen hat die Prüfung der Ge- } \\
\text { brauchstauglichkeit auch die unterhalb des Dauerstauzieles liegenden } \\
\text { Bauwerkselemente zu beinhalten. Sofern erforderlich ist hierfür der } \\
\text { Dauerstauraum zu entleeren }\end{array}$ & $\begin{array}{l}\text { In DIN 19700-12:2004-7 werden in Abweichung oder Ergän- } \\
\text { zung zu DIN 19700-11 Hinweise für Hochwasserrückhalte- } \\
\text { becken gegeben. Grundsätzlich besteht auch an Hochwasser- } \\
\text { rückhaltebecken die Erfordernis von Vertieften Überprüfungen }\end{array}$ \\
\hline \multirow[t]{2}{*}{$\begin{array}{l}\text { 19700-13: } \\
2019-6 \\
\text { Staustufen }\end{array}$} & 9.2 & $\begin{array}{l}\text { Funktionsprüfungen von Verschlüssen sind regelmäßig durchzufüh- } \\
\text { ren. } \\
\text { In größeren Zeitabständen sind die Verschlüsse, auch die schwer } \\
\text { zugänglichen Teile (ggf. unter Zuhilfenahme von Besichtigungsein- } \\
\text { richtungen, Einrüstungen u.ä.) handnah zu prüfen. Hierfür ist eine } \\
\text { Möglichkeit zur Kontrolle von Verschlussteilen, die bei normalem } \\
\text { Betrieb unter Wasser liegen, vorzusehen }\end{array}$ & \multirow[t]{2}{*}{$\begin{array}{l}\text { Diese Norm wurde } 2019 \text { in aktualisierter Form veröffentlicht. } \\
\text { 9.2.4 gibt Hinweise auf die Funktionsprüfungen der } \\
\text { Verschlüsse. Diese sind vergleichsweise häufig } \\
\text { durchzuführen. Die tiefer gehenden Überprüfungen der } \\
\text { Verschlüsse können - müssen aber nicht - den Vertieften } \\
\text { Überprüfungen zugeordnet werden. } \\
\text { In 9.4. wird ein Zeitrahmen für die Vertieften Überprüfungen } \\
\text { genannt }\end{array}$} \\
\hline & 9.4 & $\begin{array}{l}\text { In angemessenen Zeitabständen (etwa } 10 \text { Jahre bis } 20 \text { Jahre) sind im } \\
\text { Rahmen einer Vertieften Überprüfung die statischen, hydrologischen } \\
\text { und hydraulischen Bemessungsgrundlagen sowie die betrieblichen } \\
\text { Vorgaben und das Überwachungskonzept zu beurteilen }\end{array}$ & \\
\hline
\end{tabular}

Tab. 2 DVWK-Merkblatt 231/1995 „Sicherheitsbericht Talsperren-Leitfaden“; Zitate zu Vertieften Überprüfungen

\begin{tabular}{lll}
$\mathrm{Nr}$ & Relevante Formulierungen in Bezug auf vertiefte Überprüfungen (Zitat) & Anmerkung \\
5 & Vertiefte Überprüfung & Seit August 2020 wird das DVWK-Merkblatt \\
Eine vertiefte Überprüfung der Talsperre sollte etwa alle 10 Jahre sowie gegebenenfalls nach au- & 231 von der DWA-Arbeitsgruppe WW-4.8 \\
Bergewöhnlichen Ereignissen durchgeführt werden. Entspricht eine Talsperre nicht den allgemein & überarbeitet. Ein wesentlicher Aspekt ist \\
anerkannten Regeln der Technik, so hat ebenfalls eine vertiefte Überprüfung stattzufinden. & hierbei die Konkretisierung der Inhalte zur \\
Im Rahmen dieser weitergehenden Untersuchung sind die statischen, hydrologischen und hydrauli- & Durchführung der Vertieften Überprüfung. \\
schen Bemessungsgrundlagen sowie die betrieblichen Vorgaben und das Überwachungskonzept zu & Zudem soll die Anwendbarkeit auf alle Stau- \\
anberprüfen. & anlagen (Hochwasserrückhaltebecken, \\
$\begin{array}{ll}\text { Hierbei können gegebenenfalls auch statische, (hydro-)geologische oder hydrologische Gutachten } & \text { Staustufen, Pumpspeicherbecken und Sedi- } \\
\text { unter Verwendung neuer Erkenntnisse (Auswertung der vorliegenden Messreihen, neue wissenschaft- } & \text { mentationsbecken) unter Berücksichtigung } \\
\text { liche Methoden) erforderlich werden. Hieraus ergeben sich möglicherweise neue Beurteilungskriterien } & \text { der Kategorisierung hinsichtlich der Größe/ } \\
\text { für verschiedene Messwerte. } & \text { Bedeutung geprüft und ggfs. weiterentwi- } \\
\text { In einem Bericht sind die durchgeführten Untersuchungen und deren Ergebnisse zu dokumentieren. } \\
\text { Der Sicherheitszustand der Talsperre ist zu beurteilen und - falls erforderlich - muss ein Konzept für } \\
\text { notwendige Anpassungsmaßnahmen aufgestellt werden. Gegebenenfalls werden auch betriebliche } \\
\text { Vorgaben und Änderungen im Überwachungskonzept erforderlich }\end{array}$ \\
\hline
\end{tabular}




\section{G. Zusammenfassung und abschließen-} de Beurteilung.

Grundsätzlich sind in dieser Struktur die in den Regelwerken (siehe oben) genannten Themengebiete enthalten. Aufgrund der zum Zeitpunkt der Veröffentlichung bereits vorliegenden ersten praktischen Erfahrungen seit der Einführung im Jahre 1995 orientiert sich die vorgeschlagene Gliederung nun jedoch deutlich an den konkret zu untersuchenden Bauwerken.

\section{Praktische Erfahrungen und Empfehlungen}

\subsection{Erfahrungsstand}

Nach deutlich über 20 Jahren praktischer Erfahrung mit den Vertieften Überprüfungen in Deutschland werden in den nachfolgenden Ausführungen Erkenntnisse und Empfehlungen der Autoren an einer größeren Anzahl von Talsperren in verschiedenen Bundesländern Deutschlands (insbesondere in Bayern, Thüringen und NordrheinWestfalen) zusammengefasst.

\subsection{Themenbereiche, Grundlagen}

Bei der Durchführung von Vertieften Überprüfungen in Deutschland werden unter Bezugnahme auf DIN 19700-11, Abschnitt 10.4 in der Regel folgende sicherheitsrelevante Themen behandelt:

- Hochwassersicherheit,

- Zuverlässigkeit der Absperrbauwerke und Einzelbauwerke (Tragsicherheit, Gebrauchstauglichkeit, Dauerhaftigkeit),

- baulicher Zustand der Absperrbauwerke und Einzelbauwerke,

- Stabilität der Stauraumhänge,

- Bauwerksüberwachung (Mess- und Kontrollsystem, Überwachungsergebnisse),

- Unterlagendokumentation.

Die Bearbeitung der genannten Themen setzt voraus, dass alle Grundlagen, die für den sicheren Betrieb der Anlage erforderlich sind, bekannt sind. $\mathrm{Zu}$ Beginn der Vertieften Überprüfung ist ihre Aktualität zu prüfen. Diese Grundlagen können sein

- Bemessungshochwasserabflüsse $\mathrm{BHQ}_{1}$ und $\mathrm{BHQ}_{2}$,

- Stauspiegelhöhen bei planmäßigem Betrieb und im Hochwasserfall,

- meteorologische Situation, z. B. Wind (Wellenauflauf), Temperaturen
(Zwangsbeanspruchungen im Bauwerk, Exposition bei Frost), Eislast,

- Erdbebenlasten,

- Verkehrslasten (z.B. auf der Dammkrone oder auf Bermen, Schiffsanprall etc.),

- Betriebsweise der Anlage,

- Untergrundverhältnisse,

- Materialkennwerte,

- zugrundeliegende Regelwerke.

\subsection{Bauwerke und Bauteile -} Räumliche Abgrenzung

Gegenstand einer Vertieften Überprüfung sind in der Regel alle Bauwerke und Bauteile, die für die Sicherheit einer Stauanlage erforderlich sind. Eingeschlossen sind die relevanten Elemente des Stahlwasserbaus und der technischen Ausrüstung. Diese Vorgehensweise berücksichtigt die entsprechenden Passagen in Abschnitt 11 der DIN 19700-10, in dem die Zuverlässigkeitsanforderungen an Tragwerke geregelt sind. Neben dem eigentlichen Absperrbauwerk einschließlich Untergrund gilt dieser Abschnitt der Norm auch für Einzelbauwerke und -bauteile sowie für deren Staubeckenhänge und -böschungen.

\subsubsection{Talsperren und Hochwasserrückhaltebecken}

Mindestens folgende Bauwerke sind Gegenstand einer Vertieften Überprüfung:

- Absperrbauwerk,

- Grundablass einschließlich ggf. zugehöriger Stollenbauwerke und Energieumwandlung,

- Hochwasserentlastung einschließ lich ggf. zugehöriger Stollenbauwerke und Energieumwandlung,

- Stützwände etc.

Es handelt sich damit um Bauwerke, die sich im Normalfall im Umfeld des Absperrbauwerks befinden.

Bauwerke, die ausschließlich der gewerblichen Nutzung der Talsperre dienen, z.B. Triebwasserleitungen bei Kraftwerksbetrieb oder Entnahmebauwerke bei Rohwasserbereitstellung werden vom Betreiber häufig "freiwillig“ in die Beurteilung mit aufgenommen. Falls an diesen Bauwerke bei einem Schadensfall sicherheitsrelevante Folgeschäden entstehen können, so ist auch hier aus fachlicher Sicht eine Sicherheitsüberwachung erforderlich, welche jedoch nicht zwingend der Ver- tieften Überprüfung nach DIN 19700 zuzuordnen ist.

\subsubsection{Staustufen}

Mindestens folgende Bauwerke sind Gegenstand einer Vertieften Überprüfung:

- Absperrbauwerk (Wehranlage, Kraftwerksblock),

- Wehrverschlüsse,

- Tosbecken,

- Stützwände etc.,

- Stauhaltungsdämme.

Die Berücksichtigung von Triebwasserleitungen oder Kanalbauwerken kann notwendig sein, wenn bei einem Schadensfall sicherheitsrelevante Folgeschäden entstehen können.

\subsubsection{Pumpspeicherbecken}

Bei Pumpspeicherwerken können Oberbecken und Unterbecken jeweils wie eine Talsperre behandelt werden, sofern sie mit Grundablass und Hochwasserentlastungsanlage ausgestattet sind.

In Deutschland haben die Oberbecken häufig kein eigenes Einzugsgebiet, sodass dann auch meist keine Hochwasserentlastungsanlage und fallweise auch kein eigener Grundablass vorhanden sind. Die einzige Entleerungsmöglichkeit ist dann die Triebwasserleitung, die für die Sicherheit der Anlage von großer Bedeutung ist und Bestandteil der Vertieften Überprüfung sein muss. In der Praxis treten hier häufig Interessenskonflikte auf, da eine Begutachtung der Triebwasserleitung einen längeren Betriebsstillstand bedeuten kann. Die Begutachtung von Triebwasserleitungen wird dann fallweise zunächst zurückgestellt und bei der nächsten betrieblich erforderlichen Anlagenabstellung nachgeholt. Diese Vorgehensweise ist nur sinnvoll, wenn der zeitliche Abstand nicht $\mathrm{zu}$ groß wird. Teilweise müssen hier Einzelfallentscheidungen getroffen werden, bei denen auch die mögliche Schadensfolge eine Rolle spielen kann. Die Begutachtung (Begehung) von Triebwasserleitungen bedeutet meist einen hohen zeitlichen und technischen Aufwand, da die Zugänglichkeit vor allem bei steilen Rohrleitungen sehr schwierig sein kann. Hinzu kommen „Ausfallkosten“ für den entstehenden Betriebsstillstand. 


\subsection{Fachleute, Berichtswesen}

Aufgrund des weiten Themenbereichs erfolgt die Bearbeitung meist durch ein Team aus mehreren Fachleuten. Diese Fachleute müssen neben einer besonderen Fachkunde auch über allgemeine Erfahrungen auf dem Gebiet der Stauanlagen verfügen. Zur Sicherstellung der Objektivität sollte die Bearbeitung nicht durch betriebseigenes, sondern durch externes Personal erfolgen. Folgende Fachbereiche können bei der Auswahl der Fachleute relevant sein:

- Hydrologie, Hydraulik, Wasserbau,

- Massivbau,

- Stahlwasserbau,

- Grundbau, Geologie,

- Messtechnik.

Üblicherweise werden für die jeweiligen Fachbereiche eigene Fachberichte erstellt. In diesen Fachberichten wird die jeweilige Thematik ausführlich behandelt, ggf. vorhandene Defizite werden herausgearbeitet und erläutert. Hinsichtlich des baulichen Zustands kann eine Zuordnung der Bauteile in eine $\mathrm{Zu}$ standskategorie erfolgen. Bei notwendigen Maßnahmen muss ein Zeithorizont für die Abarbeitung angegeben werden.

Wichtig ist, dass in einem zusammenfassenden Prüfbericht die Ergebnisse aller Fachberichte knapp dargestellt werden. Nachfolgend wird beispielhaft die Gliederung des zusammenfassenden Prüfberichts bei der Vertieften Überprüfung einer Trinkwassertalsperre in Deutschland gezeigt (Tab. 3).

\subsection{Einbindung der Aufsichtsbehörde}

Der Rahmen der Überwachung wasserbaulicher Anlagen ist in Deutschland im Wasserhaushaltsgesetz geregelt (siehe oben). Weitergehende Regelungen finden sich in den Landeswassergesetzen und den zugehörigen Verwaltungsvorschriften der einzelnen Bundesländer. Aufgrund der föderalistischen Strukturen in Deutschland sind diese Regelungen in den Bundesländern nicht einheitlich, jedoch ist die grundsätzliche Vorgehensweise vergleichbar.

Entsprechend der relevanten Gesetze und Vorschriften sind Stauanlagen nach den allgemein anerkannten Regeln der Technik herzustellen, zu unterhalten und zu betreiben. DIN 19700 stellt hierbei eine allgemein anerkannte Regel der Technik dar. Da in dieser Norm als Teil des Sicherheitskonzeptes die Durchführung von Vertieften Überprüfungen enthalten ist, ergibt sich hieraus zwangsläufig die Verpflichtung, Vertiefte Überprüfungen durchzuführen.

Die zuständigen Behörden überwachen die Einhaltung der entsprechenden Gesetze und Vorschriften und damit auch die Durchführung einer Vertieften Überprüfung. In den einzelnen deutschen Bundesländern werden diese Behörden nicht einheitlich bezeichnet. Beispielsweise kann es sich um Wasserwirtschaftsämter, staatliche Umweltämter, Ministerien für Umwelt und Landwirtschaft oder die Bezirksregierungen handeln. Im Folgenden wird

\section{Tab. 3 Beispiel für die Gliederung eines zusammenfassenden Prüfberichtes für eine Trinkwassertalsperre in Deutschland}

\section{Teil I Allgemeine Angaben}

\section{I.1 Überblick Talsperre}

I.2 Technische Daten, Hydrologische Bemessungsgrößen

I.3 Geologie an der Sperrenstelle

I.3.1 Überblick

I.3.2 Staubecken

I.3.3 Dammgründung

I.4 Beschreibung der Talsperrenanlage

I.4.1 Absperrbauwerk

1.4.2 Hochwasserentlastung

I.4.3 Grundablass und Entnahmebauwerk

I.4.4 Messtechnische Bauwerksüberwachung

I.5 Anlagendokumentation

I.5.1 Talsperrenbuch

1.5.2 Betriebsvorschrift

I.5.3 Unterlagen zur Talsperre, Bestandspläne

I.6 Unterlagen zur Vertieften Überprüfung

Teil II Zustandsbericht Bausubstanz, Funktionsfähigkeit

II.1 Allgemeines

II.1.1 Vertiefte Überprüfung

II.1.2 Zustandskategorien

II.2 Dammbauwerk, Entwässerungseinrichtungen

II.2.1 Baulicher Zustand Dammbauwerk, Entwässerungseinrichtungen

II.2.2 Erforderliche Maßnahmen Dammbauwerk, Entwässerungseinrichtungen

II.3 Hochwasserentlastung

II.3.1 Baulicher Zustand Hochwasserentlastung

II.3.2 Erforderliche Maßnahmen Hochwasserentlastung

II.4 Entlastungs- und Entnahmebauwerk

II.4.1 Baulicher Zustand Entlastungs- und Entnahmebauwerk

II.4.2 Erforderliche Maßnahmen Entlastungs- und Entnahmebauwerk

II.5 Uferbereiche Stausee, Stauwurzel, Verlandungszustand

II.5.1 Zustand Uferbereiche, Stauwurzel, Verlandung

II.5.2 Erforderliche Maßnahmen Uferbereiche, Stauwurzel, Verlandung

II.6 Stahlwasserbau

II.6.1 Baulicher Zustand und Funktionsfähigkeit Stahlwasserbau

II.6.2 Erforderliche Maßnahmen Stahlwasserbau

II.7 Mess- und Kontrolleinrichtungen

II.7.1 Baulicher Zustand und Funktionsfähigkeit Mess- und Kontrolleinrichtungen

II.7.2 Erforderliche Maßnahmen Mess- und Kontrollsystem
Teil III Stauanlagenüberwachung und -sicherheit

III.1 Vorbemerkung

III.2 Hochwassersicherheit, Hydraulik

III.2.1 Vorbemerkung

III.2.2 Bemessungshochwasserzuflüsse

III.2.3 Hochwasserstauziele, Retentionsberechnung

III.2.4 Freibord

III.2.5 Leistungsfähigkeit der Hochwasserentlastung

III.2.6 Erforderliche Maßnahmen Hochwassersicherheit

III. 3 Zuverlässigkeitsnachweise Absperrbauwerk

III.3.1 Vorbemerkung

III.3.2 Grundlagen der Nachweise

III.3.3 Tragsicherheitsnachweise

III.3.4 Gebrauchstauglichkeitsnachweise

III.3.5 Prüfung der Zuverlässigkeitsnachweise

III.3.6 Erforderliche Maßnahmen Zuverlässigkeitsnachweise

III.4 Zuverlässigkeitsnachweise Hochwasserentlastung

III.4.1 Vorhandene Nachweise

III.4.2 Erforderliche Maßnahmen Zuverlässigkeitsnachweise

III.5 Zuverlässigkeitsnachweise Entnahmebauwerk

III.5.1 Vorhandene Nachweise

III.5.2 Erforderliche Maßnahmen Zuverlässigkeitsnachweise

III.6 Zuverlässigkeitsnachweise Stahlwasserbau

III.6.1 Vorhandene Nachweise

III.6.2 Erforderliche Maßnahmen Zuverlässigkeitsnachweise

III.7 Bauwerksüberwachung

III.7.1 Vorbemerkung

III.7.2 Beurteilung der Bauwerksüberwachung

III.7.3 Beurteilung der Messergebnisse

III.7.4 Erforderliche Maßnahmen Bauwerküberwachung 
die Bezeichnung „Aufsichtsbehörde“ verwendet.

Die Verantwortung für die rechtzeitige und regelmäßige Durchführung einer Vertieften Überprüfung liegt beim Betreiber der Anlage. Dies bedeutet, dass der Betreiber selbst für die Beauftragung der erforderlichen Fachleute und die Abwicklung der Überprüfung zuständig ist. Ebenso trägt der Betreiber alle anfallenden Kosten. Insbesondere für Stauanlagen, die dem Hochwasserschutz dienen oder andere allgemeine Aufgaben erfüllen, sind zudem enge Abstimmungen mit der Aufsichtsbehörde notwendig. Beispielsweise kann bei der Trockenlegung von Grundablässen die maximal mögliche Wasserabfuhr und damit der Betrieb im Hochwasserfall beschränkt sein.

Betreibern großer Stauanlagen oder Betreibern, die für eine Vielzahl von Stauanlagen zuständig sind, sind die entsprechenden Gesetze und Vorschriften in der Regel bekannt. Die erforderlichen Vertieften Überprüfungen werden dann meist "freiwillig“ bzw. ohne gesonderte Hinweise der Aufsichtsbehörde durchgeführt. Bei kleineren Betreibern sind häufig entsprechende Aufforderungen notwendig.

Mit der Durchführung von Vertieften Überprüfungen werden meist Ingenieurbüros beauftragt. Jedoch gibt es Ausnahmen; beispielsweise wird in Bayern bei staatlichen Wasserspeichern häufig die Vertiefte Überprüfung vom Freistaat Bayern selbst durchgeführt.

Die Ergebnisse der Vertieften Überprüfung sind der Aufsichtsbehörde vorzulegen. Diese überprüft die übergebenen Unterlagen, wobei diese Überprüfung hinsichtlich ihrer Tiefe je nach Bundesland sehr unterschiedlich sein kann.

In einigen Bundesländern werden Dokumente wie Tragsicherheitsnachweise, Windgutachten, hydraulische Berechnungen, Nachweise der Hochwassersicherheit etc. von der Aufsichtsbehörde selbst oder von hinzugezogenen externen Gutachtern oder Prüfingenieuren überprüft. Es werden dann gesonderte Prüfberichte oder Bestätigungen, teilweise auch mit zusätzlichen Auflagen erstellt und dem Betreiber übergeben. Für den Fall, dass von der Aufsichtsbehörde zusätzlich externe Gutachter hinzugezogen werden, entstehen zusätzliche Honorarkosten, die vom Betreiber getragen werden müssen. In manchen Bundesländern wird auf diese zusätzliche Kontrolle vollständig oder teilweise verzichtet.

\subsection{Zeitbedarf, Bearbeitungsaufwand}

Als Ergebnis von Vertieften Überprüfungen ergeben sich oft Defizite, die behoben werden müssen. Es kann sich hierbei um bauliche Defizite oder um Defizite hinsichtlich der vorhandenen Unterlagen handeln. Bauliche Defizite sind innerhalb eines vorzugebenden Zeitrahmens zu beheben. In der Regel werden hier zunächst weitere Planungsarbeiten erforderlich.

Bei Defiziten hinsichtlich der vorhandenen Unterlagen handelt es sich beispielsweise um notwendige Ergänzungen von Tragsicherheitsnachweisen, fehlende Gutachten o. Ä. Bei der Abarbeitung dieser „Unterlagendefizite" bestehen hinsichtlich der zeitlichen Eingliederung grundsätzlich zwei Möglichkeiten:

A. Erstellung aller notwendigen Ergänzungs-Unterlagen bereits im Rahmen der Vertieften Überprüfung.

B. Abschluss der Vertieften Überprüfung mit einer „Defizitliste“. In dieser Defizitliste werden u.a. die fehlenden und folglich $\mathrm{zu}$ erstellenden Unterlagen auflistet und ein realistischer Zeithorizont für die Erstellung vorgegeben.

Fall A hat den Vorteil, dass nach Abschluss der Vertieften Überprüfung sämtliche Unterlagen, die die Stauanlage betreffen, auf einem aktuellen Stand sind. Ein großer Nachteil ist jedoch, dass sich die Durchführung der Vertieften Überprüfung dann über mehrere Jahre hinziehen kann. In Deutschland sind Vertiefte Überprüfungen bekannt, die sich über mehr als 10 Jahre hinziehen.

Fall B hat den Vorteil, dass die Vertiefte Überprüfung in einem kompakten Zeitrahmen durchgeführt und abgeschossen werden kann. Sämtliche Defizite können und müssen im Nachgang abgearbeitet werden.

Aus Sicht der Autoren ist Fall B, also eine kompakte Durchführung der Vertieften Überprüfung, zu bevorzugen. Dies gibt dem Betreiber Sicherheit und reduziert in der Regel sowohl für die Bearbeiter als auch für den Betreiber die Kosten. Bei einer lang andauernden Bearbeitung besteht zudem die Gefahr, dass die Bearbeiter wechseln, was zu einem deutlichen Qualitätsverlust führen kann.
Aus Sicht der Autoren sollte eine Vertiefte Überprüfung einer Talsperre innerhalb eines Jahres, maximal innerhalb von zwei Jahren, abgeschlossen werden. Je nach Größe und Nutzungsart einer Stauanlage entsteht bei der Durchführung einer Vertieften Überprüfung ein unterschiedlich hoher Aufwand. Hierbei spielen u.a. die vorhandene Unterlagensituation sowie die Frage, ob es sich um eine erste Vertiefte Überprüfung oder bereits um eine Wiederholung handelt, eine wesentliche Rolle. Der Aufwand kann durchaus zwischen 2 Personenmonaten (bei guter Unterlagensituation und einfacher Stauanlage) und mehr als 30 Personenmonaten (bei komplexer Stauanlage mit schwieriger Unterlagensituation) liegen.

\subsection{Bearbeitungsgrundlagen, phasenweise Bearbeitung}

Eine wesentliche Grundlage für die Durchführung einer Vertieften Überprüfung ist das Talsperrenbuch (allg. Stauanlagenbuch). Gemäß DIN 19700 muss das Stauanlagenbuch vor allem über die Zweckbestimmung der Anlage und die konstruktive Gestaltung der Einzelbauwerke Auskunft geben sowie alle Angaben enthalten, die für die Überwachung, den Betrieb und die Unterhaltung der Stauanlage von Bedeutung sind. Im Stauanlagenbuch sollten wichtige Planunterlagen, statische und hydraulische Berechnungen, Ergebnisse der Bauwerksüberwachung etc. enthalten sein.

Fallweise erfüllen die vorhandenen Stauanlagenbücher diese Anforderungen nicht. Häufig sind sie nicht auf einem aktuellen Stand. Meist ist eine zusätzliche Archivrecherche notwendig.

Es ist deshalb nicht immer einfach, bereits zu Beginn einer Vertieften Überprüfung einen genauen Überblick über eine Stauanlage zu erhalten. Eine fundierte Festlegung der Vorgehensweise bei der Durchführung der Vertieften Überprüfung und auch eine realistische Abschätzung des erforderlichen Zeitbedarfs und des Bearbeitungshonorars sind dann schwierig.

Häufig wird eine Vertiefte Überprüfung deshalb in 2 Phasen durchgeführt. Phase I kann folgende Tätigkeiten enthalten:

- Begehung der Anlage, Startgespräch,

- Sichtung und Erfassung des Unterlagenbestandes, ggf. Archivrecherche, 


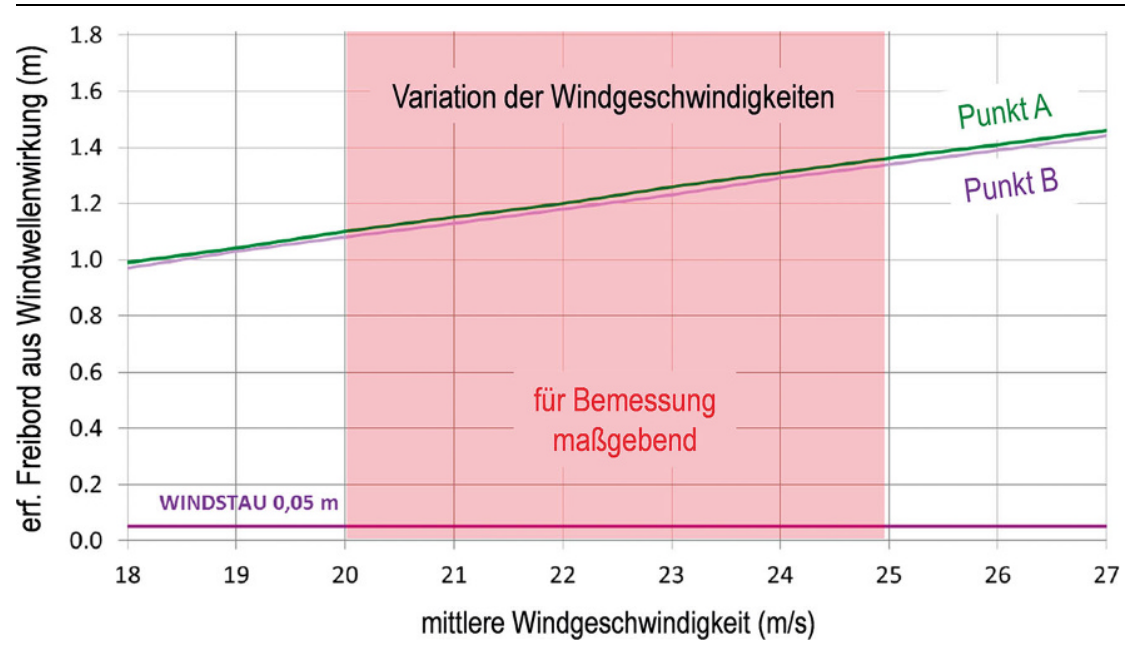

Abb. 2 Darstellung der Ergebnisse der Überprüfung des Freibords im Hochwasserbemessungsfall 1 für zwei charakteristischePunkte eines Staudamms nach DVWK-Merkblatt 246 „Freibordmessung“(1997)

- Prüfung der Unterlagen auf Vollständigkeit, erste vorläufige Beurteilung der Unterlagen,

- Zusammenstellung der sicherheitsrelevanten Bauwerke und Bauteile,

- Empfehlungen für ggf. erforderliche weitergehende Untersuchungen, soweit zu diesem Zeitpunkt bereits erkennbar (z. B. ergänzende geologische Untersuchungen, hydraulische Berechnungen etc.),

- bei Bedarf Abstimmung mit der Aufsichtsbehörde,

- Konzept für die weitere Vorgehensweise,

- Zusammenfassung der Ergebnisse in einem Bericht.

In Phase II erfolgt dann die eigentliche Überprüfung.

\section{Typische Fragestellungen und Anpassungserfordernisse bei der Vertieften Überprüfung von Talsperren}

\subsection{Grundlagendaten und Änderungen von Grundlagendaten}

In einem ersten Schritt ist es erforderlich, die für die Bauwerkstypen und weitere Strukturen aktuell geltenden Regelwerke und Normen zusammenzustellen. Im Zuge der weiteren Bearbeitung der Vertieften Überprüfung sind diese jeweils mit dem Letztstand der vorliegenden Nachweise (i.A. die vorausgegangene Vertiefte Überprüfung) $\mathrm{zu}$ vergleichen.

\subsection{Hydrologie}

Die Talsperren in Deutschland sind bereits in die Jahre gekommen. In Relation gen sich die Längen der hydrologischen Zeitreihen in einer vergleichbaren Größenordnung. Naturgemäß müssen daher auch die hydrologischen Bemessungsgrundlagen aktualisiert werden. Hieraus ergeben sich fallweise neue Bemessungshochwasserabflüsse $\mathrm{BHQ}_{1}$ und $\mathrm{BHQ}_{2}$ (nach DIN 19700). Diese aktualisierten Abflussgrößen sind wichtige Eingangsgrößen für die weiteren Nachweise.

\subsection{Wasserbau}

\subsubsection{Stauziele und Freiborde, Nachweis der Hochwassersicherheit}

Auf Basis der gegebenenfalls aktualisierten Bemessungshochwasserabflüsse $\mathrm{BHQ}_{1}$ und $\mathrm{BHQ}_{2}$ sind die Hochwasserstauziele $\mathrm{Z}_{\mathrm{H} 1}$ und $\mathrm{Z}_{\mathrm{H} 2}$ bei Bedarf anzupassen. In der Praxis hat sich gezeigt, dass in vielen Fällen - auch bei unveränderten Hochwasserstaufzielen - eine Überprüfung der vorhandenen Freiborde in den Hochwasserbemessungsfällen 1 und 2 erforderlich war. Hierbei hat es sich vielfach als sehr zweckmäßig erwiesen, eine Bandbreite der relevanten mittleren Windgeschwindigkeiten in die Auswertung miteinzubeziehen (Abb. 2). Hierdurch kann fallweise auf die mitunter sehr zeitaufwendige Anforderung eines aktualisierten Windgutachtens verzichtet werden. zu ihrer bisherigen Lebensdauer bewe-
Der Nachweis der Hochwassersicherheit ist ein zentraler Punkt der Vertieften Überprüfung. In die hierfür erforderliche Bewertung gehen die hydrologischen und hydraulischen Überprüfungen (Leistungsfähigkeit der Hochwasserentlastungsanlage, siehe unten) und der Vergleich der vorhandenen mit den erforderlichen Freibordmaßen ein.

\subsubsection{Hydraulik}

Im Zuge der Vertieften Überprüfung sind die hydraulischen Nachweise der Betriebseinrichtungen (i.A. Hochwasserentlastungsanlage und Entnahmeanlagen) auf ihre Aktualität hin zu bewerten und gegebenenfalls anzupassen (z. B. bei Änderung der Grundlagendaten oder bei Defiziten in der bisherigen Nachweisführung). Dies kann durch das Prüfen der vorliegenden Nachweise, durch Nachrechnung mit geeigneten empirischen Formeln oder anhand numerischer Berechnungen erfolgen. In Einzelfällen können auch physikalische Modelle oder Erprobungen vor Ort (Abb. 3) zweckmäßig und notwendig sein.

\subsubsection{Anordnung und betriebliche Erfordernisse der Betriebseinrichtungen}

Es ist zu überprüfen, inwieweit die Hochwasserentlastungsanlage sowie die Entnahmeeinrichtungen dem Stand der Technik entsprechen. Typische Fragestellungen und Aspekte dieser Prüfung sind:

- Anordnung und Betriebssicherheit der Hochwasserentlastungsanlage (einschl. Verklausungsgefahr),

- Anwendung der (n-1) bzw. (n-a)Regel (u.a. Überprüfung der beim Nachweis der Hochwassersicherheit getroffenen Annahmen, s. oben),

- Revisionsverschlüsse (insbesondere Überprüfung und Bewertung der Revisionskonzepte und der damit verbundenen zeitweisen Blockaden von Abflussquerschnitten),

- Entleerungsmöglichkeit (Grundablass mit ausreichender Leistungsfähigkeit),

- Anordnung der Verschlussorgane an Grundablassleitungen (in der Regel 2 Verschlüsse hintereinander),

- Möglichkeit des zuverlässigen Absperrens von Entnahmeeinrichtungen (z. B. Triebwasserwege) bei etwaigen Schadensfällen, 
- Sicherheit gegen Überschreiten der höchsten zulässigen Wasserspiegel (insbesondere bei Pumpspeicherbecken).

Beispielsweise sind im Zuge einer Vertieften Überprüfung auch die etwaige Existenz und tatsächliche Handhabung von Verriegelungsmechanismen an Stauklappen zu untersuchen und zu bewerten (Abb. 4). Dies kann hinsichtlich der (n-a)-Nachweise von erheblicher Bedeutung sein, da ein Ablegen der Klappen ohne Fremdenergie dann fallweise nicht möglich ist.

\subsection{Absperrbauwerk, Dichtungssystem und Bauwerksüberwachung}

\subsubsection{Absperrbauwerk}

Im Vorfeld und in Ergänzung zu den statischen Nachweisen (siehe Abschn. 4.5) wird der grundsätzliche „sichtbare“ Zustand des Absperrbauwerks insbesondere durch detaillierte Begehungen und das Einbeziehen der Dokumentation der routinemäßigen visuellen Kontrollen (d.h. unter Verwendung der jährlichen Sicherheitsberichte) eingehend untersucht. Typische Ergebnisse dieser visuellen Prüfungen sind die Lokalisierung potenzieller Problemzonen (z.B. durch Hinweise auf Vernässungen) oder auch das Feststellen unzulässiger bzw. ungünstiger Entwicklungen im Bauwerksbereich (z. B. Bewuchs auf Staudämmen).

\subsubsection{Dichtungssystem von Bauwerk und Untergrund}

Das Gesamtsystem des Dichtungssystems ist hinsichtlich seines grundsätzlichen Entwurfes, potenzieller Schwachstellen (z. B. in den Anschlussbereichen zwischen Untergrundabdichtung und Dichtungssystem des Absperrbauwerkes) und seines Zustands zu untersuchen bzw. zu bewerten. Hierzu sind die vorliegenden Unterlagen, die Ergebnisse der Begehungen (siehe oben) und der Bauwerksüberwachung (siehe unten) heranzuziehen. Dieser Aspekt der Bauwerksüberwachung ist von besonderer Bedeutung. Insbesondere die Dichtungssysteme der Absperrbauwerke unterliegen einer systembedingt mehr oder weniger ausgeprägten $\mathrm{Al}$ terung. Ein etwaiges Sanierungserfordernis muss im Zuge der Vertieften Überprüfungen erkannt werden. Vergleichsweise häufig wird als Prüfergeb-

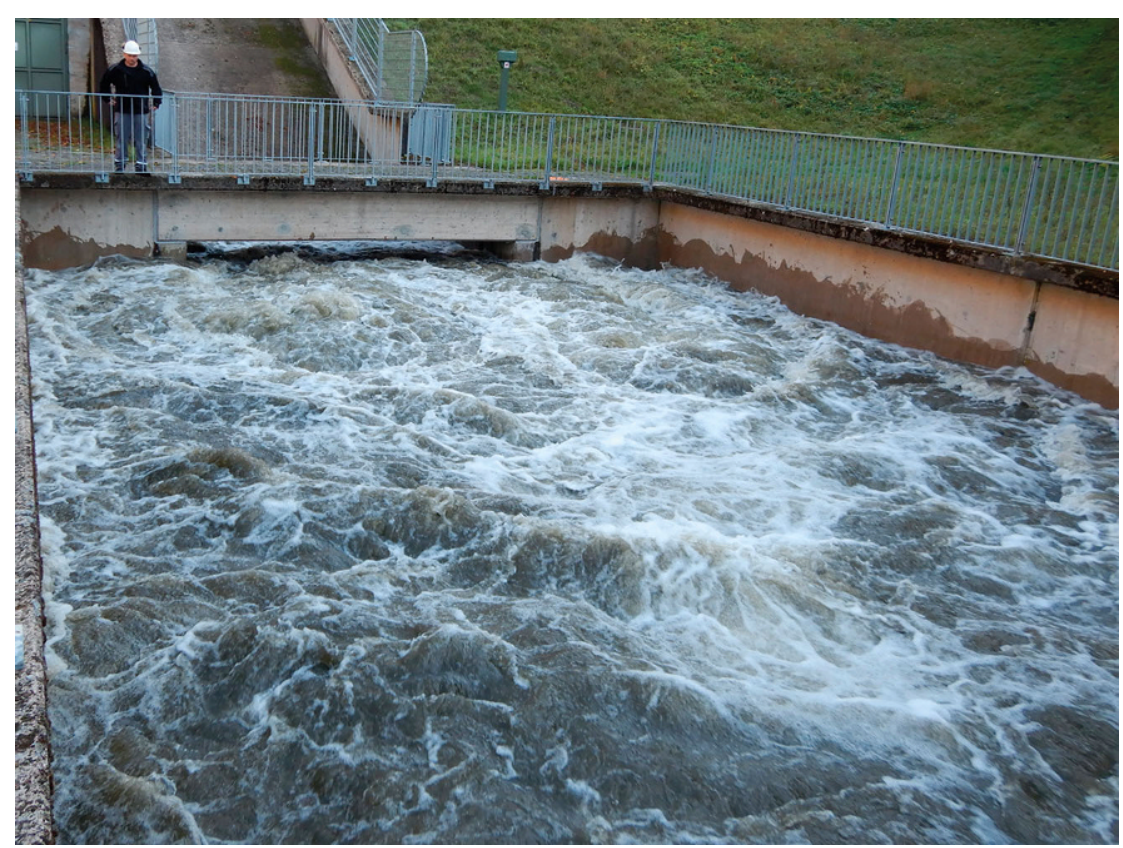

Abb. 3 Überprüfung der Energieumwandlung im Tosbeckens eines Grundablasses einer Talsperre
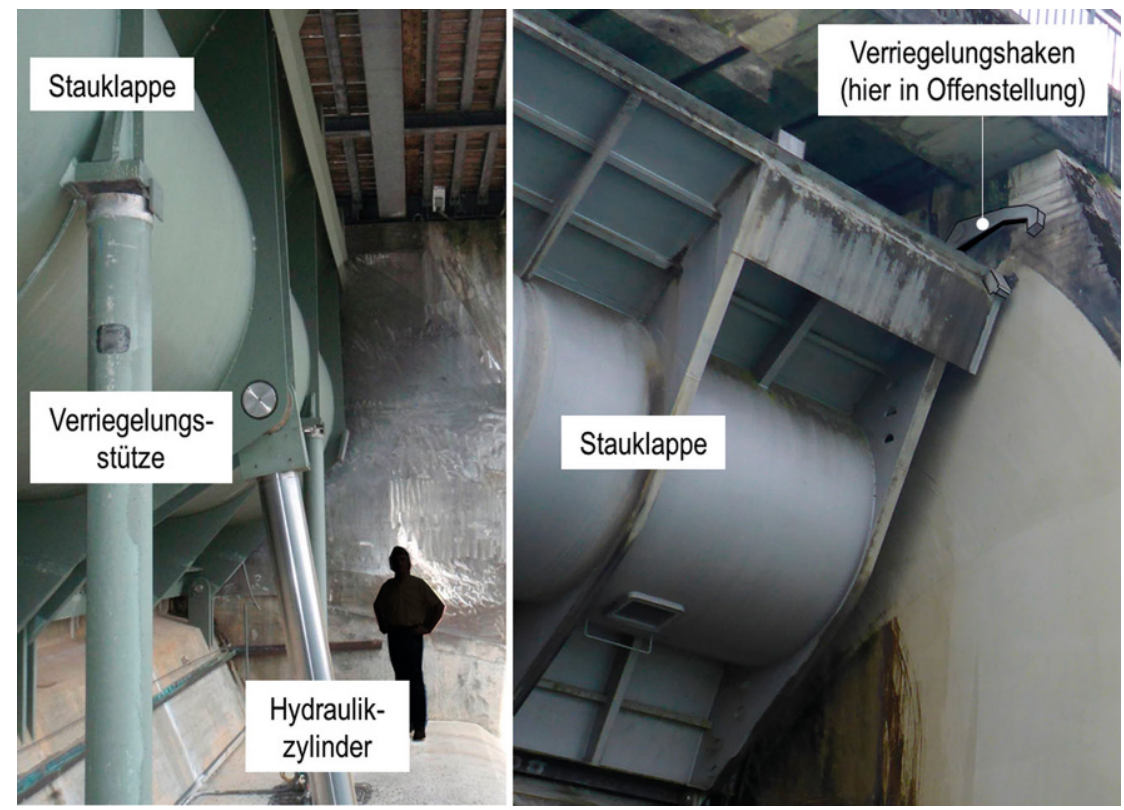

Abb. 4 Verriegelungsmechanismen an Stauklappen-u. U. relevant bei (n-a)-Nachweisen

nis ein grundsätzlich sich verstärkender Sanierungsbedarf beschrieben und ein entsprechender Hinweis für die nächste Überprüfung vermerkt.

\subsubsection{Bauwerksüberwachung}

Der Umfang, die messtechnische Qualität und die Art der Durchführung der routinemäßigen Bauwerksüberwa- chung sind $\mathrm{zu}$ bewerten. Wichtigste Basis hierfür sind die Ergebnisse aus den jährlichen Sicherheitsberichten. Durch weitergehende Auswertungen können gegebenenfalls wichtige Erkenntnisse gewonnen werden, welche über die Interpretation in den jährlichen Sicherheitsberichten hinausgehen. Dies könnte z.B. das Erstellen von Differenzenplänen bei hydraulischen 


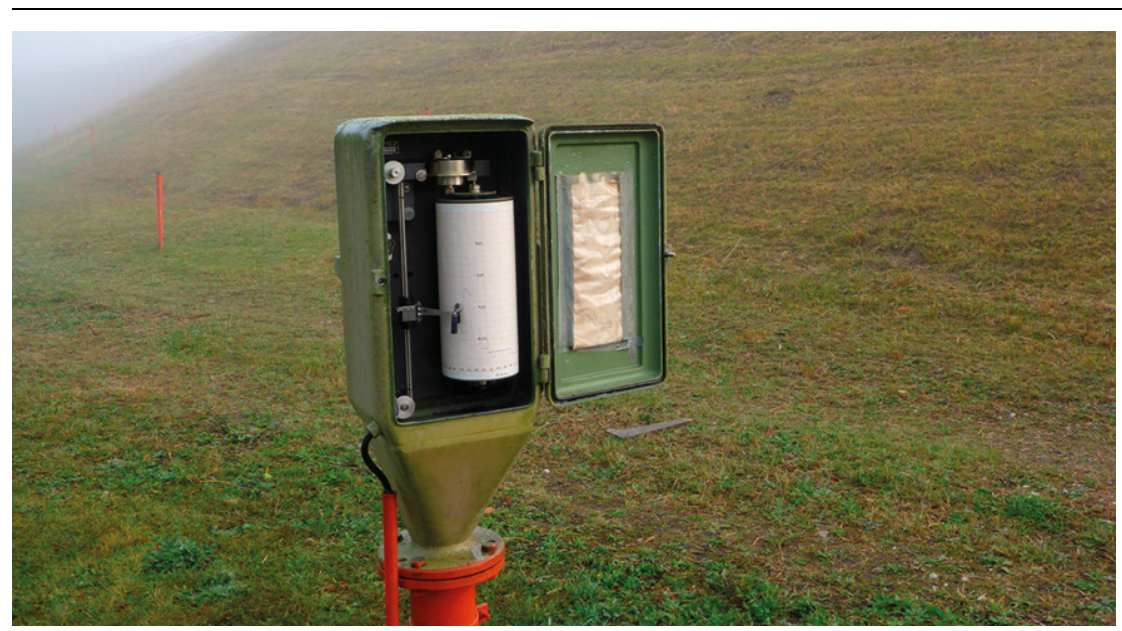

Abb. 5 Sehr robuster, aber nur mehr eingeschränkt verwendbarer (keine Fernübertragung, keine Digitalisierungsmöglichkeit) Schreibpegel einer Wasserstandmessung in einem Pegelrohr an der Luftseite eines Staudamms

Messungen oder die Darstellung sehr langer Reihen von Sickerwasserabflussmessungen als Ganglinien sowie in Abhängigkeit von der Stauhöhe sein. Ungünstige Entwicklungen müssen im Zuge dieser tief gehenden Auswertungen erkannt werden.

Wichtig ist es aus Sicht der Autoren auch, im Zuge der Vertieften Überprüfung den Umfang der Bauwerksüberwachung kritisch zu bewerten. Typische Fragestellungen sind hierbei:

- Entspricht die Bauwerksüberwachung dem Stand der Technik (vgl. Merkblatt DWA-M 514 „Bauwerksüberwachung an Talsperren“ (2011))?

- Wie können ungünstige Entwicklungen (insbesondere eine Zunahme der Durchströmung des Dichtungssystems) rechtzeitig erkannt werden?

- Welche Messungen sind von besonderer Relevanz?

- Ist es erforderlich und vorteilhaft, die automatisierte Erfassung von Bauwerksmessungen und die Fernübertragung $\mathrm{zu}$ verstärken (Beispiel Abb. 5)?

- Ist es möglicherweise sinnvoll, den Umfang der Messungen aufgrund fehlender Aussagekraft der Messergebnisse zu reduzieren?

- Ist die Nachrüstung mit modernen integralen Messverfahren sinnvoll (z.B. stationäre Laserscanstationen, verteilte faseroptische Temperaturmessungen zur Leckageortung)?

- Werden die Ergebnisse der visuellen Kontrollen ausreichend gut und nachvollziehbar dokumentiert?
- Ist die Ablage der Messergebnisse einfach und gut nachvollziehbar organisiert?

\subsection{Massiv- und Dammbauwerke}

\subsubsection{Vorbemerkung}

Wesentliche Bearbeitungsschwerpunkte bei der Überprüfung und Beurteilung von Massiv- und Dammbauwerken sind nen Zuverlässigkeitsnachweise sowie der bauliche Zustand.

\subsubsection{Grundlagensituation}

Folgende Grundlagen betreffen unmittelbar die Massiv- und Dammbauwerke:

- Geologische Situation, Untergrundkennwerte,

- Dammbaumaterialien mit Kennwerten,

- Materialkennwerte Massivbau, z.B. Mauerwerk oder Beton,

- weitere Materialkennwerte wie Dichtungsmaterialien (Asphalt, Kunststoffdichtungsbahn etc.).

Zunächst erfolgt eine Überprüfung der $\mathrm{zu}$ diesen Grundlagen vorhandenen Informationen und Unterlagen hinsichtlich Vollständigkeit und Plausibilität und auch hinsichtlich vorhandener Widersprüche. Die Ergebnisse der Überprüfungen werden in einer entsprechenden Beurteilung zusammengestellt. Sofern hier Defizite vorliegen, kann die Durchführung ergänzender Untersuchungen oder Erkundungen die Grundlagensituation, die vorhande- notwendig sein (z. B. geotechnische Erkundung, Mauerwerksprüfungen etc.).

\subsubsection{Zuverlässigkeitsnachweise}

Ein Hauptkriterium zur Beurteilung der Sicherheit einer Anlage ist der rechnerische Nachweis der Zuverlässigkeit. Der Umfang der erforderlichen Nachweise ist in DIN 19700 geregelt. Dieser Nachweis betrifft die

- Tragsicherheit,

- Gebrauchstauglichkeit und

- Dauerhaftigkeit.

Im Rahmen einer Vertieften Überprüfung werden hinsichtlich der Zuverlässigkeitsnachweise folgende Kriterien überprüft:

- Vollständigkeit der vorhandenen Nachweise,

- Aktualität der vorhandenen Nachweise.

Bezüglich der Aktualität der Nachweise wird beispielsweise überprüft:

- Entsprechen die Nachweise den aktuellen Regelwerken?

- Wurden evtl. bauliche Veränderungen berücksichtigt?

- Entsprechen die Lastannahmen den aktuellen Kenntnissen (Stauspiegelhöhen, Eislast, Erdbebenlast etc.)?

- Entsprechen die Materialkennwerte und Untergrundkennwerte den aktuellen Kenntnissen?

Die Ergebnisse der Überprüfungen werden wiederum in einer entsprechenden Beurteilung zusammengestellt. Sofern die Aktualität der vorhandenen Nachweise nicht gegeben ist, kann eine Ergänzung bzw. Neuerstellung von Nachweisen erforderlich sein. Hierbei ist allerdings eine pragmatische Vorgehensweise wichtig. Sofern beispielsweise für die prüfenden Fachleute offensichtlich ist, dass die vorhandenen Zuverlässigkeitsnachweise trotz gewisser Unstimmigkeiten „auf der sicheren Seite liegen“, ist eine Neuerstellung der Nachweise nicht notwendig.

\subsubsection{Baulicher Zustand}

Vorgehensweise Die Begutachtung des baulichen Zustands erfolgt visuell und sofern möglich „handnah“.

Ziel muss sein, alle sicherheitsrelevanten Bauteile $\mathrm{zu}$ begutachten. Dies gilt auch für die schwer zugänglichen Bauteile. Beispielsweise können bei stollenartigen Entlastungsbauwerken 
für die notwendigen Trockenlegungen sehr aufwendige Vorbereitungsmaßnahmen notwendig sein. Da eine Vertiefte Überprüfung aber genau darauf abzielt, eine Anlage sehr detailliert zu prüfen, kann hierauf normalerweise nicht verzichtet werden.

Sofern Trockenlegungen nicht möglich sind, kann die Überprüfung ggf. auch mit Tauchern erfolgen. Bei großen Wasserspiegelhöhen, z. B. an der Wasserseite von Staumauern oder in gefährlichen Bereichen, sind allerdings auch einer Überprüfung mit Tauchern Grenzen gesetzt. Eine unmittelbare Begutachtung ist dann nicht möglich. Sofern aufgrund der laufenden Bauwerksüberwachung keine Anzeichen für Schäden im Bereich nicht überprüfbarer Bauwerksbereiche vorliegen, kann dies akzeptiert werden. Entsprechende Anzeichen wären beispielsweise erhöhte Sickerwasserabflüsse, sich ändernde Sohlen- oder Porenwasserdrücke oder Unregelmäßigkeiten bei den Verschiebungsmessungen. Umso größerer Bedeutung kommt deshalb einer funktionierenden Bauwerksüberwachung zu.

Wichtiger Bestandteil eines Absperrbauwerks ist die Untergrundabdichtung. Da eine visuelle Überprüfung nicht möglich ist, kommt auch hier der Bauwerksüberwachung eine besondere Bedeutung zu.

Mindestens folgende Bauwerksbereiche sollten einer vollständigen visuellen Begutachtung unterzogen werden:

- Luftseite, Wasserseite, Krone von Absperrbauwerken,

- Hohlräume, z. B. Kontrollgänge, Schieberkammer etc.,

- Dränageeinrichtungen wie Sickerschächte, Sickerleitungen, Dammfußdränage etc.,
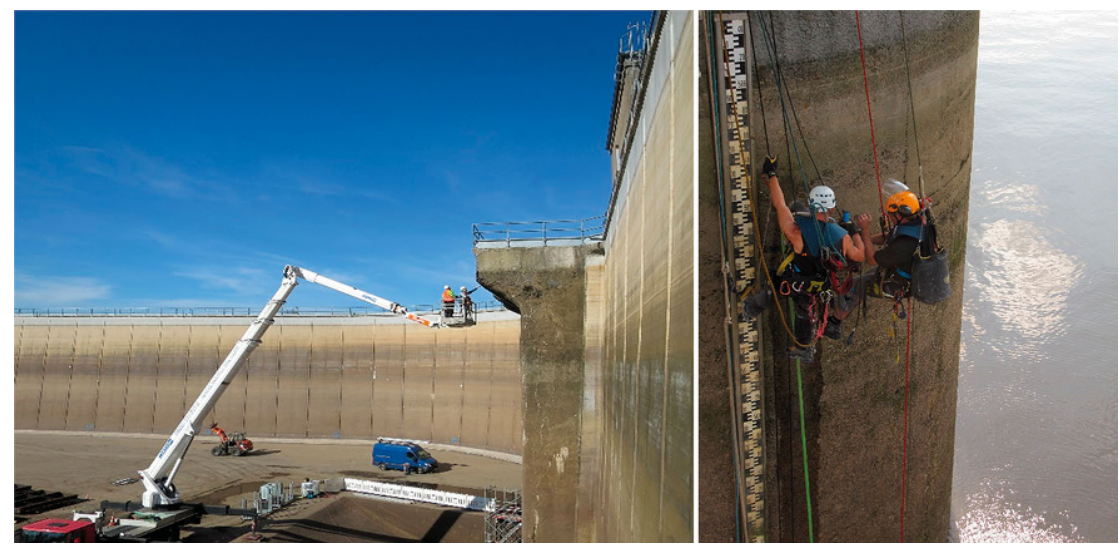

Abb. 6 Begutachtung mittels Hubsteiger bzw. Seilsicherung
Folgende visuell erkennbare Schäden oder andere Auffälligkeiten werden bei Vertieften Überprüfungen in Deutschland vergleichsweise häufig dokumentiert:

- Bruchsteinmauerwerk, z. B. bei einer

Staumauer:

- Wasserdurchtritte an der Luftseite, im Winter „Frostbärte“,

- Wasserdurchtritte in Hohlräumen, z.B. Kontrollgang,

- Schäden an der Verfugung,

- Schäden im Bereich von Fugen (falls vorhanden),

- Bewuchs.

- Betonbauteile:

- Risse, mit oder ohne Wasserführung, teilweise mit Versinterungen,

- Bewuchs aus Rissen,

- Frostschäden,

- Schäden an Fugen,

- Schäden infolge Karbonatisierung,

- Hohllagen,

- Treiberscheinungen (AKR, Sulfattreiben),

- Geschädigte Abdichtung im Kronenbereich.

- Dammbauwerke:

- Bewuchs mit Bäumen,

- Erosion im Bereich der Böschungen,

- Schäden an der wasserseitigen Schutzschicht,

- Wühltierbefall,

- Wasseraustritte, Vernässungen, bereichsweise andersartiger $\mathrm{Be}$ wuchs bei Durchfeuchtung.

- Dichtungssysteme:

- Asphaltbeton: abgewitterte Mastix, Risse, Aufbrüche/Blasen, Fehlstellen,

- Beton: siehe oben,

- Oberflächige Lehmdichtung o. Ä.: fehlende Schutzschicht, Löcher, Risse,

- Kunststoffdichtungsbahn: Risse, Schäden im Stoßbereich.

- Stauraumhänge und andere Böschungen, Stauraum:

- Gefahr von Rutschungen (in Deutschland gering),

- Gefahr von Windwurf mit Eintrag von Bäumen,

- Ufererosion,

- Verlandung (u.U. relevante Reduzierung des Hochwasserschutzraums).

Zudem kann auch Vandalismus mit unspezifischen Schäden oder Auffälligkeiten nie ausgeschlossen werden. 


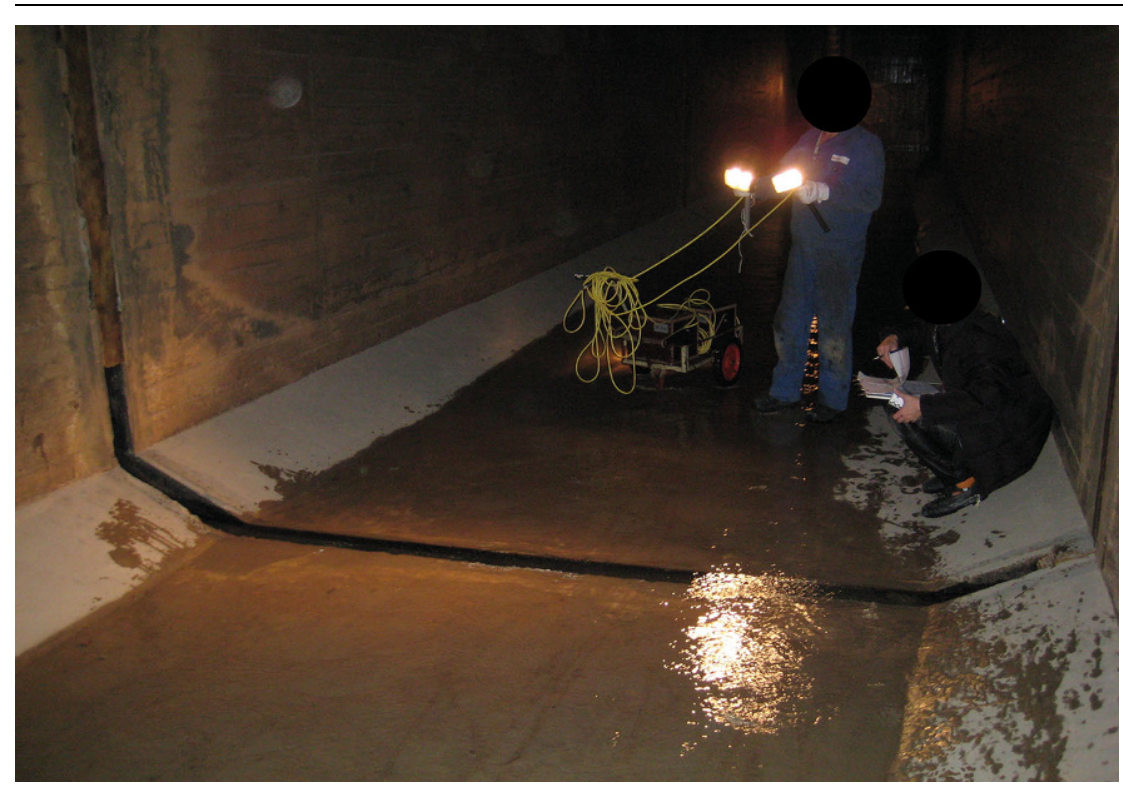

Abb. 7 Fehlende Fugenfüllung im Sohlbereich eines Grundablassstollens

Abb. 7 zeigt eine schadhafte Fuge in einem Grundablassstollen, der quer durch einen Staudamm führt. Der Grundablassstollen wurde nur für die Begehung im Rahmen der Vertieften Überprüfung trockengelegt. Diese Trockenlegung war sehr aufwendig, da die Revisionsverschlüsse nur von der Dammkrone mit einem Autokran mit knapp $40 \mathrm{~m}$ Ausleger und zusätzlichem Tauchereinsatz gesetzt werden konnten. Die Begutachtung hat ergeben, dass ein Großteil aller Fugen zum Teil stark geschädigt war. Aufgrund der Bedeutung des Grundablasses für die Sicherheit der gesamten Anlage folgt eine Instandsetzung der Fugen.

Bei der Ringmauer eines Staubeckens sind durchwegs Ausblühungen

\subsection{Stahlwasserbau}

\subsubsection{Funktionelle Einheiten}

Bei Anlagen des Stahlwasserbaus handelt es sich in der Regel um bewegliche Verschlussorgane, mit denen Ab- und Durchflussöffnungen abgesperrt, geöffnet oder reguliert werden können. Sie sind wesentliche und meist sicherheitsrelevante Anlagenbestandteile, die jeweils eine funktionelle Einheit bilden. Diese funktionellen Einheiten, die alle dem übergeordneten Begriff Stahlwasserbau zugeordnet werden, können in vier Untergruppen aufgeteilt werden (Abb. 9).

Das Ziel einer Vertieften Überprüfung ist es, die Betriebssicherheit dieser funktionellen Einheiten unter den aktuellen Gegebenheiten und Betriebsbedingungen zu überprüfen und zu hinterfragen sowie Defizite aufzuzeigen, zu bewerten und Risiken abzuschätzen.

Die hierbei praktizierte Vorgehensweise lässt sich aufgrund der Unterschiedlichkeit und Vielfalt der jeweiligen Anlagen nicht durch ein allgemeingültiges Schema definieren. Wichtige und in der Praxis immer wiederkehrende Bearbeitungsabläufe lassen sich aber durchaus mit einer strukturierten Vorgehensweise beschreiben. Es hat sich bewährt, sich im Vorfeld zu den eigentlichen Funktionsprüfungen einen umfassenden Überblick über die vorhandenen Dokumente und bautechnischen Unterlagen zu verschaffen. Diese werden in der Regel vom Betreiber zur Verfügung gestellt und müssen dann gesichtet, systematisch zusammengestellt und hinsichtlich Relevanz und Aktualität bewertet werden. Vor allem
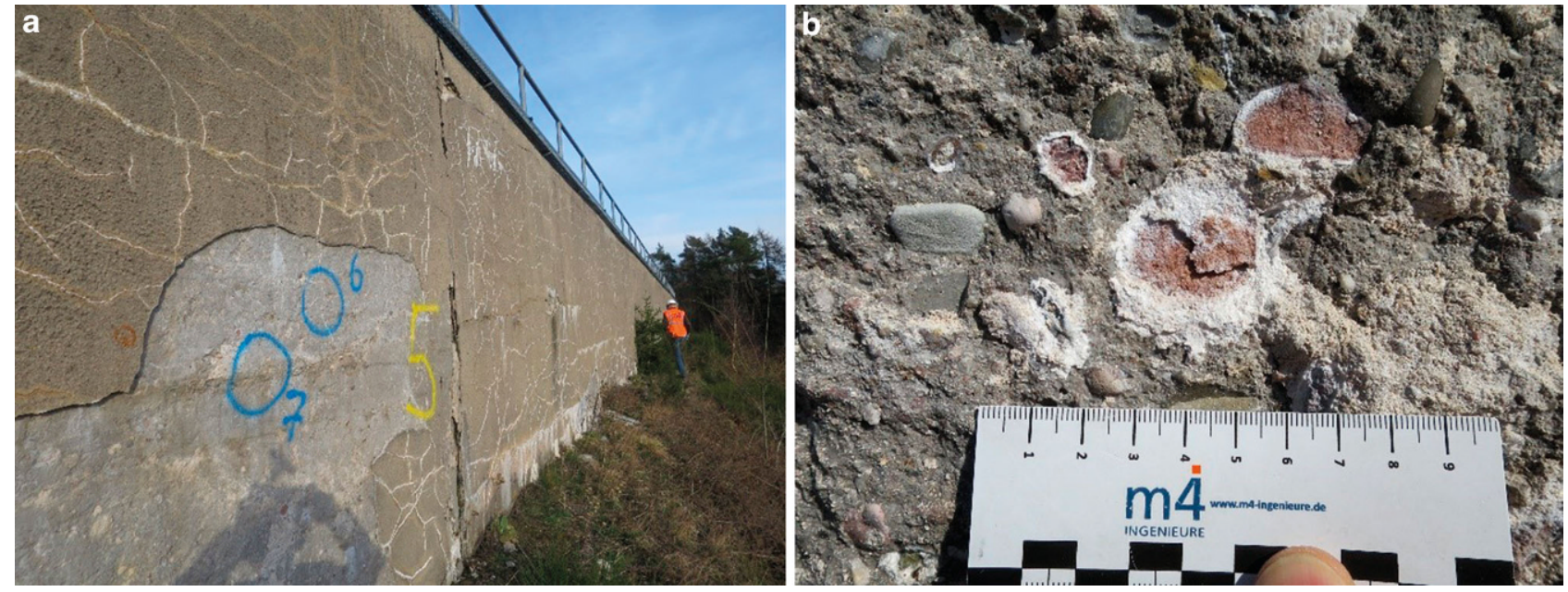

Abb. 8 Betonoberfläche mit Ausblühungen (a), Ausblühungen an den Zuschlagstoffen (b) 

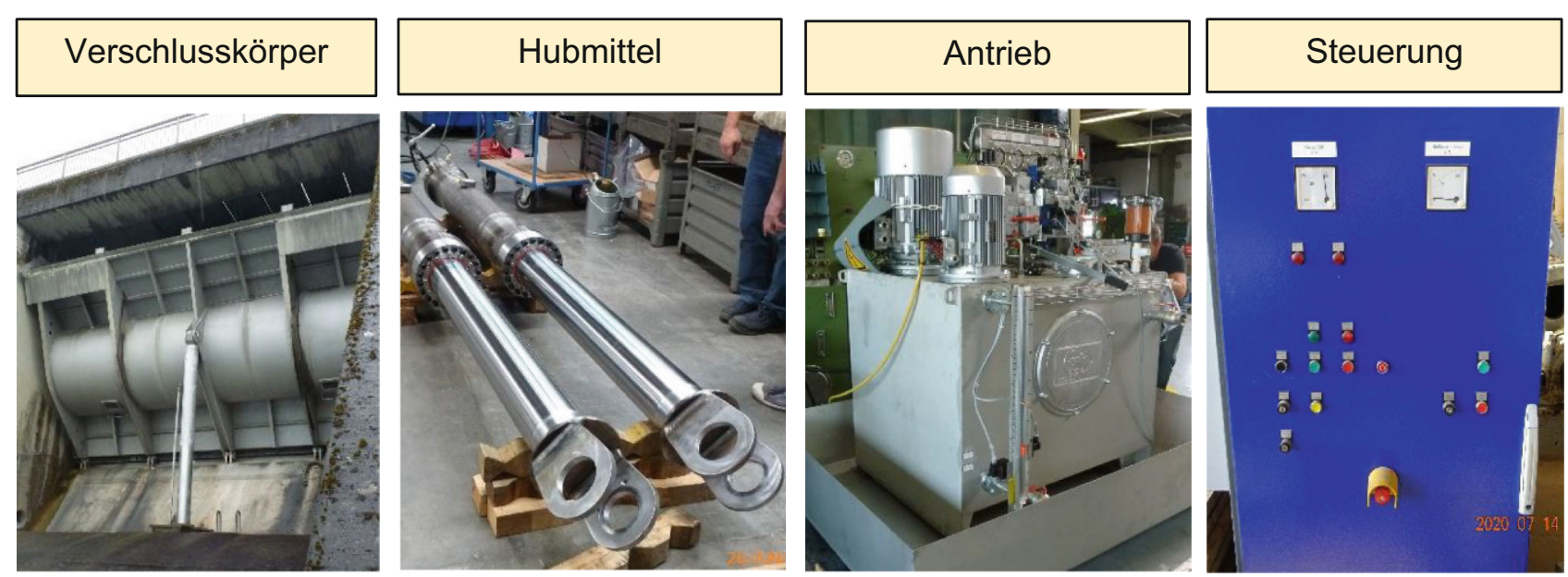

Abb. 9 Komponenten einer funktionellen Einheit des Stahlwasserbaus am Beispiel einer ölhydraulisch angetriebenen Klappe

Tab. 4 Praxisbeispiel zu einem mit dem Betreiber abzustimmenden Ablaufplan

\begin{tabular}{|c|c|c|c|c|c|c|}
\hline Lage & Gegenstand & Prüfungen & Prüfumfang & Vorbereitung/Zugänglichkeit & $\begin{array}{l}\text { Dauer } \\
\text { [Std.] }\end{array}$ & Termin \\
\hline \multirow{13}{*}{$\begin{array}{l}\text { HW- } \\
\text { Entlastung/ } \\
\text { Trockenprü- } \\
\text { fungen }\end{array}$} & \multirow{11}{*}{$\begin{array}{l}\text { Fischbauchklappe mit } \\
\text { ölhydr. } \\
\text { Torsionsrohrantrieb }\end{array}$} & \multirow{11}{*}{$\begin{array}{l}\text { Sicht- und } \\
\text { Trockenprü- } \\
\text { fungen bei } \\
\text { Wasserstand } \\
\text { Grundsee }\end{array}$} & $\begin{array}{l}\text { Sichtprüfung der Stauwandseite } \\
\text { (Klappe gelegt) }\end{array}$ & \multirow{11}{*}{$\begin{array}{l}\text { Grobe Vorreinigung der } \\
\text { Verschlussflächen, Beistellung Leiter. } \\
\text { Beleuchtung des Arbeitsbereichs auf der } \\
\text { Rückseite des Verschlusses. } \\
\text { Bedienung des Antriebs durch } \\
\text { geschultes Personals }\end{array}$} & \multirow[t]{11}{*}{2,00} & \multirow[t]{20}{*}{ Termin 2} \\
\hline & & & $\begin{array}{l}\text { Sichtprüfung der Seitenbleche und } \\
\text { Klappenlager }\end{array}$ & & & \\
\hline & & & $\begin{array}{l}\text { Sichtprüfung der Klappen-Rückseite } \\
\text { (Klappe gestellt) }\end{array}$ & & & \\
\hline & & & $\begin{array}{l}\text { Sichtprüfung des Torsionsrohran- } \\
\text { schlusses und der Stopfbuchse }\end{array}$ & & & \\
\hline & & & $\begin{array}{l}\text { Korrosionsschutz mit Schichtdicken- } \\
\text { messung }\end{array}$ & & & \\
\hline & & & $\begin{array}{l}\text { Sichtprüfung Torsionsrohrhebel und } \\
\text { Zylinderanschluss }\end{array}$ & & & \\
\hline & & & Kontrolle Endschaltereinstellung & & & \\
\hline & & & $\begin{array}{l}\text { Sichtkontrolle Zylinder, Stellungsge- } \\
\text { ber und Verrohrung }\end{array}$ & & & \\
\hline & & & Sichtkontrolle der Klappenbelüftung & & & \\
\hline & & & $\begin{array}{l}\text { Sicht- und Funktionskontrolle Hy- } \\
\text { draulikaggregat und Steuerschrank }\end{array}$ & & & \\
\hline & & & $\begin{array}{l}\text { Funktionsprüfung Handpumpe und } \\
\text { Druckbegrenzung }\end{array}$ & & & \\
\hline & \multirow[t]{2}{*}{$\begin{array}{l}\text { Treibgutabweiser } \\
\text { (Grobrechen) }\end{array}$} & \multirow{2}{*}{$\begin{array}{l}\text { Sichtprüfung } \\
\text { bei } \\
\text { Wasserstand } \\
\text { Grundsee }\end{array}$} & $\begin{array}{l}\text { Sichtprüfung der } 9 \text { Abweisträger } \\
\text { (Hohlträger) mit den Sohl- und } \\
\text { Kopfauflagern }\end{array}$ & \multirow{2}{*}{$\begin{array}{l}\text { Der Zustand wird visuelle vom Boden } \\
\text { des Einlaufs und von der oberen } \\
\text { Betriebsebene aus beurteilt. Gerüste } \\
\text { oder Leitern sind nicht erforderlich }\end{array}$} & \multirow[t]{2}{*}{0,50} & \\
\hline & & & $\begin{array}{l}\text { Sichtprüfung Zustand des Korrosi- } \\
\text { onsschutz }\end{array}$ & & & \\
\hline \multirow[t]{7}{*}{$\begin{array}{l}\text { Betriebsauslass } \\
\text { Nassprüfungen }\end{array}$} & Dammtafel & \multirow{7}{*}{$\begin{array}{l}\text { Sicht-, } \\
\text { Dichtheits- } \\
\text { und Funkti- } \\
\text { onsprüfungen } \\
\text { der unter } \\
\text { Wasserdruck } \\
\text { stehenden Be- } \\
\text { triebsauslass- } \\
\text { Druckrohrlei- } \\
\text { tung }\end{array}$} & $\begin{array}{l}\text { Sichtprüfung der Dammtafelflächen } \\
\text { (Lagerplatz) }\end{array}$ & \multirow{7}{*}{$\begin{array}{l}\text { Dammtafel ausheben und vertikal } \\
\text { aufstellen. Herstellen der } \\
\text { Zugänglichkeiten und Beleuchtung der } \\
\text { Inspektionsbereiche. } \\
\text { Bedienung der Anlage durch geschultes } \\
\text { Personal }\end{array}$} & \multirow[t]{7}{*}{4,00} & \\
\hline & $\begin{array}{l}\text { Absperrklappe DN } \\
1.200 \text { mit Antrieb }\end{array}$ & & $\begin{array}{l}\text { Sicht- und Funktionsprüfung der } \\
\text { Absperrklappe und des Antriebs }\end{array}$ & & & \\
\hline & $\begin{array}{l}\text { Füllleitung mit Arma- } \\
\text { turen }\end{array}$ & & $\begin{array}{l}\text { Sicht- und Funktionsprüfung der } \\
\text { Belüftungseinrichtungen }\end{array}$ & & & \\
\hline & $\begin{array}{l}\text { Druckrohrleitung DN } \\
1.200\end{array}$ & & $\begin{array}{l}\text { Sichtprüfung der Druckrohrleitung } \\
\text { (ca. } 25 \text { längs- und rundnahtge- } \\
\text { schweißte Rohrschüsse) }\end{array}$ & & & \\
\hline & \multirow{3}{*}{$\begin{array}{l}\text { Kegelstrahlschieber } \\
\text { DN } 1.200 \text { mit Antrieb }\end{array}$} & & Sichtprüfung der ca. 25 Sattellager & & & \\
\hline & & & $\begin{array}{l}\text { Korrosionsschutz mit Schichtdicken- } \\
\text { messung }\end{array}$ & & & \\
\hline & & & $\begin{array}{l}\text { Sicht- und Funktionsprüfung des } \\
\text { Kegelstrahlschiebers }\end{array}$ & & & \\
\hline
\end{tabular}


bei einer Erstprüfung ist es hilfreich, hierzu ein tabellarisches Unterlagenverzeichnis anzufertigen.

\subsubsection{Fragestellungen im Zuge der Dokumentenrecherche}

- Haben sich die hydraulischen Randbedingungen im Laufe der Betriebsjahre geändert (z.B. Stauzielerhöhungen)?

- Hat sich die Betriebsweise der Verschlüsse geändert (z. B. Automatisierung, Pegelsteuerung etc.)?

- Wo bestehen gegebenenfalls Abweichungen zur aktuellen DIN 19704: 2014-11 (2014) und wurden Themen wie Stabilität, Gebrauchstauglichkeit, Erdbeben etc. berücksichtigt?

- Sind die Bemessungsansätze der vorliegenden Nachweise vollständig und noch aktuell?

- Wurden die in der letzten Vertieften Überprüfung festgestellten Defizite behandelt bzw. beseitigt?

- Welche relevanten Unterhaltsmaßnahmen wurden bisher durchgeführt und wurden konstruktive Änderungen dokumentiert und geprüft?

\subsubsection{Abstimmung und Vorbereitung der Prüfungen}

Für die Vorbereitung und Organisation der Sicht- und Funktionsprüfungen ist eine enge Abstimmung mit dem Betreiber erforderlich. Bereits im Vorfeld und mit genügend Vorlauf zu den Prüfaktivitäten vor Ort ist es hilfreich, einen mit allen Beteiligten abgestimmten, detaillierten Ablaufplan aufzustellen. Ein solcher Ablaufplan kann u.a. folgende Themen enthalten:

- Lage und Art des zu untersuchenden Bauteils,

- Art der Prüfung: Sicht-, Trockenoder Nassprüfungen,

- erforderliche Sondergutachter (Schadstoffanalysen, Materialproben, Vermessung u.a.),

- Beschreibung des vorgesehenen Prüfumfangs,

- Festlegung der durch den Betreiber zu veranlassenden Vorbereitungen,

- ungefähre Dauern der Untersuchungen,

- Terminfestlegungen.

Ein solcher Ablaufplan lässt sich übersichtlich und in tabellarischer Form darstellen (Tab. 4).

Die oben aufgezeigten Abstimmungen und Dokumentenrecherchen sowie

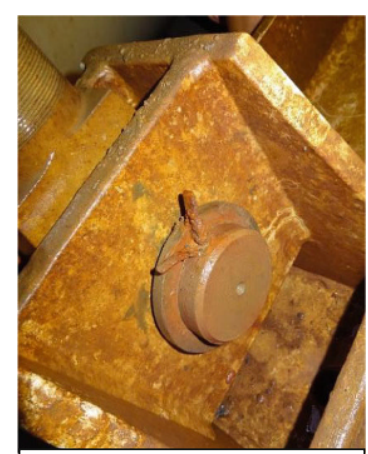

Fehlerhafte Achssicherung

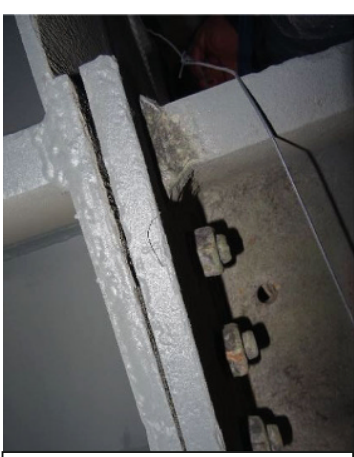

Deformierter

Segmentarmanschluss

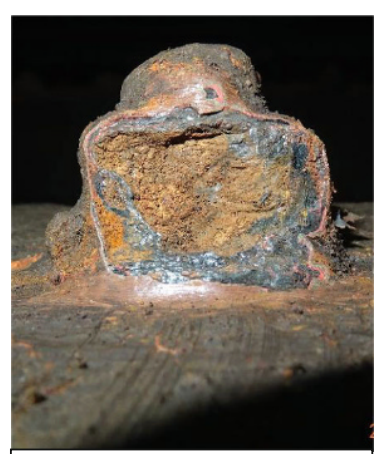

Korrodierte

Schraubverbindung

Abb. 10 Beispiele typischer und nur im Zuge einer Trockenprüfung festzustellender Defizite und Schäden

die Vorbereitungen der Prüfprotokolle und des Prüfungsablaufs können vor allem bei einer Erstprüfung einen erheblichen Arbeitsaufwand bedingen. Eine sorgfältige und gewissenhafte Bearbeitung dieser Phase 1 „Projektgrundlagen und Abstimmungen“ ist Voraussetzung und Grundlage für den weiteren Ablauf der Vertieften Überprüfung des Stahlwasserbaus.

\subsubsection{Hinweise und Empfehlungen zur Durchführung der Prüfungen}

Im Gegensatz zu den formellen, jährlich zu erstellenden Sicherheitsberichten, für die in der Regel ausschließlich die ordnungsgemäßen Funktionen der Anlagen getestet werden, muss bei der Vertieften Überprüfung der Gesamtzustand der Verschlusskörper und Antriebseinheiten beurteilt werden. Dies erfordert eine mitunter sehr aufwendige Vorgehensweise, bei der die Verschlusseinheiten zur Inaugenscheinnahme trockengelegt werden müssen. Eine Beurteilung der Betriebssicherheit hängt nicht alleine davon $a b$, ob der Verschluss zum Zeitpunkt der Prüfung seine Dienste ordnungsgemäß erfüllt und alle sicherheitsrelevanten Notfunktionen und Redundanzen bestätigt werden können. Oftmals sind es die kleineren, unauffälligen Defizite, die im Notfall und unter Höchstbeanspruchung zu einem Versagen des Gesamtsystems führen können. Die Zylinderaufhängung eines Hochdruckschützes, die nicht mit Achshaltern sondern nur über einen Splint gesichert wird, ist hierzu ein Beispiel aus der Praxis (Abb. 10). Die Gefahr, dass eine solche Achssicherung korrodiert und sich der Bolzen der Zylinderauf- hängung infolge hoher dynamischer Einwirkungen löst, ist groß und kann in Folge dazu führen, dass das sicherheitsrelevante Verschlusssystem trotz aller Redundanzen ohne vorherige Ankündigung vollständig manövrierunfähig wird.

Der Aufwand zur Durchführung von Sicht- und Trockenprüfungen ist aufgrund der erforderlichen Vorbereitungen (Revisionsverschlüsse setzen, Wasserhaltung, Zugänge, Sicherungsmaßnahmen etc.) zwar hoch, er ist aber für die Feststellung von „versteckten“ Defiziten unverzichtbar. Die Durchführung der Sicht- und Funktionsprüfungen in situ, mit den Trocken- und Nassprüfungen, kann als Phase 2 bezeichnet werden. Hierbei empfiehlt es sich, zunächst die Trockenprüfungen durchzuführen und die sicherheitsrelevanten Verschlussfunktionen zu testen. Sollten sich bei den Trockenprüfungen Probleme ergeben haben oder massive Defizite festgestellt worden sein, muss das zunächst vorgesehene Prüfprogramm der Nassprüfungen unter Umständen angepasst werden. Die Blanko-Prüfprotokolle zu den Trocken- und Nassprüfungen sollten bereits in der Phase 1 mit dem Betreiber abgestimmt werden. Im Zuge der Nassprüfung wird ein Ergebnisprotokoll erstellt (Tab. 5). Diesem Protokollbeispiel ist lediglich der durchgeführte Prüfungsumfang mit der jeweiligen Defizitfeststellung JA oder NEIN zu entnehmen. Um den Istzustand zu beschreiben und die Defizite zu erläutern, müssen die Prüfprotokolle durch Fotos und Beschreibungen vervollständigt werden. 
Tab. 5 Beispiel eines Ergebnisprotokolls in Form einer „Checkliste“

\begin{tabular}{|l|l|l|l}
\hline & & Defizite \\
Ja & Nein \\
\hline 1 & Unterschütz (US) aus Schließstellung ca. 10cm öffnen (vor Ort) & $\square$ & $\square$ \\
\hline 3 & Kontrolle Pumpendruck und Belüftung beim Anheben US & $\square$ & $\square$ \\
\hline 4 & Unterschütz wieder schließen (aus Warte) & $\square$ & $\square$ \\
\hline 5 & Kontrolle Funktion Stellungsgeber und Druckbegrenzung US & $\square$ & $\square$ \\
\hline 6 & US mit Handbedienung am Steuerblock leicht Anheben & $\square$ & $\square$ \\
\hline 7 & US mit Umschalteinheit komplett öffnen/Öffnungszeit/Drücke & $\square$ & $\square$ \\
\hline 8 & US schließen (vor Ort)/Schließzeit/Drücke & $\square$ & $\square$ \\
\hline 9 & US mit Handpumpe leicht anheben und gleich wieder schließen & $\square$ & $\square$ \\
\hline 10 & Sichtkontrollen Ölstand und Ölstandüberwachung & $\square$ & $\square$ \\
\hline 11 & Dichtigkeit Verschluss, Druckdeckel und Stangendurchführungen & $\square$ & $\square$ \\
\hline 12 & Bedienungsanweisung liegt vor Ort aus/Betriebspersonal geschult & $\square$ & $\square$ \\
\end{tabular}

\subsubsection{Auswertungen und Berichtserstellung}

Die Ergebnisse der Phase 2 werden dann zusammen mit den Ergebnissen aus der Phase 1 sowie den Ergebnissen ggf. hinzugezogener Gutachter (Schadstoffanalysen wie Asbest/PAK, Materialanalysen, Taucheruntersuchungen u. a.) in einer Defizitliste erfasst, hinsichtlich möglicher Schadensursachen analysiert und durch Empfehlungen zur Reduzierung der Risiken ergänzt. $\mathrm{Zu}$ diesem Zeitpunkt befinden wir uns bereits in der abschließenden und wichtigsten Phase 3 „Auswertungen und Berichterstellung“. Der abschließende Fachbericht Stahlwasserbau sollte in etwa folgenden Gliederungsumfang beinhalten:

- Prüfungsumfang, Vorgehensweise und Systematik,

- Beschreibung der aktuell bestehenden Anlage,

- Kontrolle der Lastannahmen und Bemessungsansätze,

- Kontrolle der technischen Unterlagen und vorhandenen Anlagendokumentation,

- Ergebnisprotokolle der Sicht- und Trockenprüfungen,

- Ergebnisprotokolle der Nass- und Funktionsprüfungen,

- Zusammenfassung der Ergebnisse mit Defizitlisten, Risikoabschätzungen und Empfehlungen.

\subsection{Betrieb}

Auch jene betrieblichen Aspekte, welche für die Sicherheit der Stauanlage relevant sind, sind Gegenstand der Vertieften Überprüfung. Besonders häufig sind hier die Existenz, die Verfügbarkeit, die Struktur und die Aktualität folgen- der grundlegender Unterlagen zu überprüfen bzw. zu kommentieren:

- Sicherheitsbericht für Talsperren Teil A (Allgemeine Angaben),

- Sicherheitsbericht für Talsperren Teil B (Jährliche Beurteilungen),

- Betriebsvorschrift (Zusammenstellung der Grundlagen der Betriebsund Bauwerksüberwachung) einschließlich des Mess- und Kontrollprogramms,

- Talsperrenbuch bzw. Stauanlagenbuch.

Von einer besonderen Bedeutung ist im Zuge der Vertieften Überprüfung der direkte fachliche Kontakt des Prüfteams mit dem Fachpersonal an der Talsperre. Die Vertrautheit mit den Bauwerken und das Detailwissen des Anlagenpersonals sind sicherheitsrelevante Aspekte. Durch die Einführung des DWAMerkblatts 1003 im Jahre 2019 liegt nun auch eine wichtige Grundlage zur Überprüfung der Qualifikation des Personals an den Talsperren vor (DWA 2019).

\subsection{Ergebnis und Umsetzung}

Das Ergebnis der Vertieften Überprüfung wird in Berichtsform zusammengestellt. Hierbei ist die Feststellung, dass die Talsperre dem Stand der Technik entspricht, von zentraler Bedeutung. Bei allen Vertieften Überprüfungen, an welchen die Autoren bisher eingebunden waren, ist diese Aussage jedoch mit Bedingungen bzw. mit Anpassungserfordernissen verbunden gewesen. Typischerweise werden diese fachlichen Empfehlungen bzw. Auflagen mit einem Zeithorizont verbunden.

\section{Fazit}

Die Vertieften Überprüfungen der Talsperren in Deutschland sind ein wesentlicher und unverzichtbarer Baustein der Sicherheitsüberwachung.

Im Auftrag, auf Kosten und in der Verantwortung des Betreibers wird ein externes Gutachter-Team mit der eingehenden Prüfung aller sicherheitsrelevanten Aspekte einer Talsperre betraut. Diese Maßnahme ist zeit- und kostenintensiv. Es besteht der Anspruch, dass alle relevanten Nachweise (Hydrologie, Wasserbau, Statik, Stahlwasserbau, Betrieb) geprüft und auf ihre Aktualität hin untersucht werden.

Typischerweise wird im Zuge dieser Prüfungen der Anstoß für etwaige Sanierungen und Modernisierungen gegeben. Es besteht der Anspruch, dass die gesamte Anlage eingehend auf alle sicherheitsrelevanten Aspekte untersucht wird und dass etwaige Defizite zuverlässig erkannt werden. Alle Beteiligten einer Vertieften Überprüfung müssen mit großer Sorgfalt und hohem Verantwortungsbewusstsein tätig sein. Die Eigenverantwortung des Betreibers spiegelt sich in der Organisation und Durchführung der Vertieften Überprüfungen wider.

Funding Open access funding provided by University of Innsbruck and Medical University of Innsbruck.

Open Access Dieser Artikel wird unter der Creative Commons Namensnennung 4.0 International Lizenz veröffentlicht, welche die Nutzung, Vervielfältigung, Bearbeitung, Verbreitung und Wiedergabe in jeglichem Medium und Format erlaubt, sofern Sie den/die ursprünglichen Autor(en) und die Quelle 


\section{Originalarbeit}

ordnungsgemäß nennen, einen Link zur Creative Commons Lizenz beifügen und angeben, ob Änderungen vorgenommen wurden.

Die in diesem Artikel enthaltenen Bilder und sonstiges Drittmaterial unterliegen ebenfalls der genannten Crea- tive Commons Lizenz, sofern sich aus der Abbildungslegende nichts anderes ergibt. Sofern das betreffende Material nicht unter der genannten Creative Commons Lizenz steht und die betreffende Handlung nicht nach gesetzlichen Vorschriften erlaubt ist, ist für die oben aufgeführten Weiterverwendungen des
Materials die Einwilligung des jeweiligen Rechteinhabers einzuholen.

Weitere Details zur Lizenz entnehmen Sie bitte der Lizenzinformation auf http://creativecommons.org/licenses/ by/4.0/deed.de.

\section{Literatur}

DIN 19700-10:2004-7 Stauanlagen: Gemeinsame Festlegungen; DIN 19700-11:2004-7 Stauanlagen: Talsperren; DIN 19700-12:2004-7 Hochwasserrückhaltebecken; DIN 19700-13:2019-

6. Stauanlagen: Staustufen. Normenausschus Wasserwesen (NAW) im DIN Deutschen Institut für Normung E. V., Beuth Verlag, Berlin.

DIN 19704-1:2014-11 Stahlwasserbauten - Teile 1 bis 3: Berechnungsgrundlagen. Normenausschuss Wasserwesen (NAW) im DIN Deutschen Institut für Normung E. V., Beuth Verlag, Berlin. DVWK (1995): Merkblatt DVWK-M 231/1995 Sicherheitsbericht Talsperren-Leitfaden. Regelwerk der Deutschen Vereinigung für Wasserwirtschaft, Abwasser und Abfall e.V. (DWA).

DVWK (1995): Merkblatt DVWK-M 246/1997.

Freibordbemessung an Stauanlagen. Regel- werk der Deutschen Vereinigung für Wasserwirtschaft, Abwasser und Abfall e. V. (DWA).

DWA (2011): Merkblatt DWA-M 514. Bauwerks überwachung an Talsperren. Regelwerk der Deutschen Vereinigung für Wasserwirtschaft, Abwasser und Abfall e. V. (DWA).

DWA (2019): Merkblatt DWA-M 1003. Anforderung an die Qualifikation von Personal an Talsperren und großen Stauanlagen. Regelwerk der Deutschen Vereinigung für Wasserwirtschaft, Abwasser und Abfall e. V. (DWA).

International Commission on Large Dams (ICOLD) (2018): Bulletin 158. Dam Surveillance Guide. Paris.

Overhoff, G., A. Bieberstein, V. Bettzieche (2004): Erfahrungen und Hinweise zum Sicherheitsbericht und zur vertieften Überprüfung von
Talsperren. Wasserwirtschaft 94 (2004) 07-08. Friedrich Vieweg \& Sohn Verlagsgesellschaft mbH, Wiesbaden

Hinweis des Verlags Der Verlag bleibt in Hinblick auf geografische Zuordnungen und Gebietsbezeichnungen in veröffentlichten Karten und Institutsadressen neutral. 